\title{
On the Feasibility of Wireless Shadowing Correlation Models
}

\author{
Sebastian S. Szyszkowicz, Student Member, IEEE, Halim Yanikomeroglu, Member, IEEE, and \\ John S. Thompson, Member, IEEE
}

\begin{abstract}
There is emerging interest in more detailed models for wireless shadowing, which may include nonconstant shadowing variance, non-lognormal shadowing, and, most importantly, correlation between paths; we focus on this last aspect. This paper offers a structured synthesis of the existing literature on autocorrelation and cross-correlation in wireless shadowing and attempts to fill existing gaps in the analysis of correlation models. We make a survey of these models and argue, as has previously been observed, that certain models are not mathematically feasible, which may lead to problems in simulations or analysis. We then state some theorems that test whether the models are positive semidefinite, which is the central necessary condition for feasibility, and evaluate the existing models accordingly. Additionally, we evaluate the models according to their physical plausibility, which leads us to choose one model among many as arguably the best one in existence so far. This paper should be useful as a guide on how to implement shadowing correlation in one's work, how to choose an appropriate correlation model, and how to modify existing models or create new models so that they fulfill mathematical feasibility.
\end{abstract}

Index Terms-Correlation, wireless channel modeling, wireless shadowing.

\section{INTRODUCTION}

C ORRELATION in wireless shadowing is a significant step in obtaining more realistic channel propagation models. This paper proposes to discuss in detail the existing literature on shadowing correlation and the existing correlation models, focusing on evaluating the mathematical feasibility of these models while also making comments on their physical plausibility. The purpose of this paper is to facilitate the choice and implementation of correlation models in works that involve shadowing.

\section{A. Motivation}

Recent work on various aspects of wireless communications has indicated a wide gap between results obtained assuming independent shadowing paths and between those that introduce correlation in shadowing propagation models [1]-[5]. Older

Manuscript received February 16, 2010; revised May 31, 2010; accepted August 24, 2010. Date of publication September 30, 2010; date of current version November 12, 2010. This work was supported in part by a $P G S D$ from the National Sciences and Engineering Research Council of Canada. The review of this paper was coordinated by Prof. T. Kuerner.

S. S. Szyszkowicz and H. Yanikomeroglu are with the Broadband Communications and Wireless Systems Centre, Department of Systems and Computer Engineering, Carleton University, Ottawa, ON K1S 5B6, Canada (e-mail: sz@sce.carleton.ca; halim@sce.carleton.ca).

J.S. Thompson is with the School of Engineering and Electronics, University of Edinburgh, EH8 9YL Edinburgh, U.K. (e-mail: John.Thompson@ed.ac.uk).

Digital Object Identifier 10.1109/TVT.2010.2082006 works show that shadowing correlation significantly affects handover behavior [6]-[9], interference power [10]-[14] (and consequently system performance) [15]-[17], and the performance of macrodiversity schemes [10], [18]-[23]. Furthermore, shadowing in decibels has been measured [18], [24]-[39] to have significant correlation in various scenarios. Because of these two facts, we believe that the general wireless community is becoming convinced of the importance of modeling correlation in shadowing [2]-[5], [12], [23], [40]-[49]. This is part of a more general trend suggesting that most propagation models used in simulation (and analysis) work today are sometimes too simplistic and may lead to misleading results [50]. Shadowing correlation can also be positively exploited in some algorithms or protocols, e.g., for wireless positioning [2], [5], [51], cognitive radio and spectrum sensing [49], [52]-[54], or neighbor discovery [48] applications.

Meanwhile, there already exist several models for shadowing correlation. The questions of which model to use and how to simulate channel realisations accordingly are essential for every researcher wanting to implement correlation. However, because correlation models are based on estimating a complex phenomenon from a small data sample, they may lose some properties with respect to reality; in particular, some estimated models may not be mathematically feasible, and thus, it would be impossible, in certain cases, to generate Monte Carlo samples from them.

In [45], it is deplored that this problem is rarely taken into account; yet, it has received some attention in [6], [7], [45], [46], and [55]-[57]. It has previously been addressed [43], [57], [58] by slightly modifying particular correlation matrices so that they become feasible (specifically, positive semidefinite ( $p s d$ ) for lognormal shadowing). However, we prefer to address the problem at its root: let us use only such correlation models that will always produce $p s d$ correlation matrices, as suggested in [6], [45], and [56]. This has the following advantages:

1) a faster implementation time, as no provision for correcting non- $p s d$ matrices needs to be made;

2) probably a faster execution time for every realisation, as no decisions or corrections need to be made during the Monte Carlo simulation;

3) mathematical consistency and elegance: if a model is feasible, then it is safe for mathematical analysis [9]-[11], [13], [21], [22], [44], [52], [59]-[64].

If we begin with a set of independent random variables and construct correlated shadowing variables by combining them in some way, then we can always calculate their correlation 
structure. However, the inverse operation cannot always be performed: a construction to obtain a desired correlation structure does not always exist. Those that cannot be constructed are termed not feasible.

\section{B. Mathematical Tools}

Some mathematical definitions and properties will be necessary in this paper. All algebraic operations are taken over the $\mathbb{R}$ field.

Definition 1: A square matrix $\mathbf{A}_{N \times N}$ is positive definite if for every column vector $\mathbf{x} \in \mathbb{R}^{N} \backslash \mathbf{0}, \mathbf{x}^{\mathrm{T}} \mathbf{A} \mathbf{x}>0[65$, p. 82, Def. 3.1.1.xvii].

Definition 2: A square matrix $\mathbf{A}_{N \times N}$ is $p s d$ if for every column vector $\mathrm{x} \in \mathbb{R}^{N}, \mathrm{x}^{\mathrm{T}} \mathbf{A} \mathrm{x} \geq 0$ [65, p. 82, Def. 3.1.1.xv].

Definition 3: The Hadamard (also called Schur) product of two $M \times N$ matrices (A with entries $a_{i, j}$ and $\mathbf{B}$ with entries $\left.b_{i, j}\right)$ is a $M \times N$ matrix $\mathbf{C}=\mathbf{A} \circ \mathbf{B}$ with entries $c_{i, j}$ such that the matrix entries are multiplied elementwise: $c_{i, j}=a_{i, j} b_{i, j}$ [65, p. 252, eq. (7.3.1)].

Property 1 (Schur Product Theorem): If two $N \times N$ matrices $\mathbf{A}$ and $\mathbf{B}$ are both $p s d$, then their Hadamard product $\mathbf{C}=\mathbf{A} \circ \mathbf{B}$ is also psd [65, p. 335, Fact 8.16.8].

Definition 4: The Kronecker (also called Zehfuss) product of a $M \times N$ matrix $\mathbf{A}$ with entries $a_{i, j}$ and a $K \times L$ matrix $\mathbf{B}$ is a $M K \times N L$ matrix $\mathbf{C}=\mathbf{A} \otimes \mathbf{B}$ with $M \times N$ block entries $a_{i, j}$ B [65, p. 248, Def. 7.1.2].

Property 2: If $\mathbf{A}$ and $\mathbf{B}$ are both $p s d$, then so is $\mathbf{C}=\mathbf{A} \otimes \mathbf{B}$ [65, p. 254, Fact 7.4.15].

Definition 5: An even function $f(t)$ is said to be positive definite if and only if for any $N$, for any $t_{1}, \ldots, t_{N}$ on $\mathbb{R}^{+}$, the matrix with entries $a_{i, j}=f\left(\left|t_{i}-t_{j}\right|\right)$ is $p s d$. This can directly be derived from [66, p. 58, eq. (1.29)] and Definition 2.

Property 3 (Bochner's Theorem): A continuous even function $f(\tau)$ is positive definite if and only if there exists a nondecreasing bounded function $F(\omega), \omega \in \mathbb{R}^{+}$such that [66, p. 92, eq. (2.53)]

$$
f(\tau)=\int_{0}^{\infty} \cos \omega \tau d F(\omega) .
$$

Property 4: Every covariance matrix is necessarily $p s d^{1}$ and symmetric [66, p. 15, eq. (0.36)].

Property 5 (Pólya's Theorem): A bounded even function $f(\tau)$ with $f(\infty)=0$ that is concave (up) on $(0, \infty)$ is necessarily positive definite [66, p. 136]. (The converse is not true.)

\section{Structure of This Paper}

In Section II, we discuss the different types of physical situations that lead us to discuss correlation in shadowing before focusing on autocorrelation and cross-correlation. In Section III, we define correlation in general mathematical terms. In Section IV, we define positive semidefiniteness, which

\footnotetext{
${ }^{1}$ Reference [56] says "positive definite," but this is too restrictive, whereas [66] also says "positive definite," but this is a difference in nomenclature, and clearly, $p s d$ is meant.
}

is the primary property to determine feasibility, and give some indications on how this property can be proved. In Section V, we discuss why models derived from real-world measurements may nevertheless prove not feasible. In Section VI, we make a comprehensive study of the correlation models in the literature and summarised these models' properties in a table. In Section VII, we impose further conditions on correlation models by talking about their physical plausibility. These physical properties are also summarised in the table, which allows us to formulate a best model that meets both mathematical and physical criteria. In Section VIII, we discuss the relationship between feasibility and positive semidefiniteness in the cases of both lognormal and non-lognormal shadowing and discuss how to generate correlated jointly lognormal shadowing. We conclude in Section IX with practical guidelines for using shadowing correlation models in one's work.

This paper represents a synthesis of existing knowledge and some new contributions. As such, all non-original statements are given appropriate references.

\section{TYPES OF CORRELATION}

In wireless communications, shadowing is a phenomenon that corresponds to a random variability of the power gain over a (directed) propagation path. A transmitting node radiates a radio signal toward a receiver node. The receiver may receive a desired signal or an interfering signal. Shadowing represents the variability of the logarithm of the received power around its expected value: we denote this quantity $S$, with $\operatorname{VAR}\{S\}<$ $\infty$. The small-scale effect of fading has been removed in the spatial dimension by averaging over a local region [45] of about 50 wavelengths [56], [62], that is, approximately $8-34 \mathrm{~m}$ for classic cellular channels [18], [24], [25], [27], [29], [37], [38].

Consider two directed paths $\overline{X_{1} Y_{1}}$ and $\overline{X_{2} Y_{2}}$ with shadowing values $S_{1}$ and $S_{2}$, respectively. Their correlation coefficient is defined as

$$
\rho_{1,2}=\frac{\mathbb{E}\left\{S_{1} S_{2}\right\}}{\sqrt{\mathbb{V A R}\left\{S_{1}\right\} \mathbb{V A R}\left\{S_{2}\right\}}} .
$$

In general, correlation may exist for any set of four points $X_{1} \neq$ $Y_{1}, X_{2} \neq Y_{2}$. However, we usually examine correlation under more specific scenarios.

\section{A. Scenarios}

Shadowing correlation can occur in various particular scenarios, specifically in peer-to-peer links [2], [67], [68], in indoor, cross-floor, and indoor-outdoor links [27], [30], and in satellite-ground communications [31], [69]-[71]. It should be noted however that most of the literature on correlation in shadowing is driven by cellular (usually urban) scenarios, where the base station/mobile and uplink/downlink dualities apply. In cellular communications, the distinction between autocorrelation and cross-correlation applies [45]. Cross-sector correlation has also been considered [72].

An important consideration for satellite and indoor scenarios is that propagation is usually considered in three, not just two, Cartesian dimensions. In this paper, we will analyze 2-D 

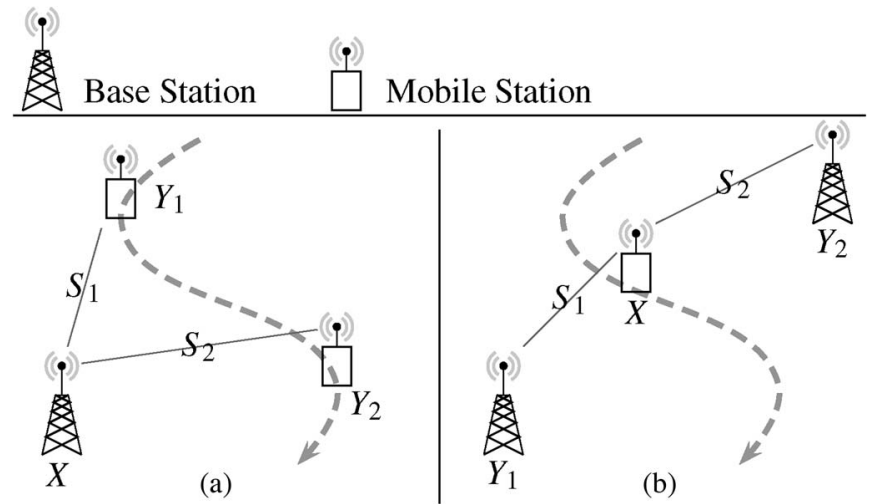

Fig. 1. (a) Shadowing autocorrelation for a mobile $Y(t)$. (b) Shadowing cross-correlation for a mobile $X$.

channel models only, although the analysis usually translates easily to three dimensions.

\section{B. Autocorrelation}

Called serial correlation [73], this model considers a transmitting base station $X$ received by the same moving mobile $Y$ at two moments in time $t_{1}$ and $t_{2}$ and at distinct locations $Y_{1}=Y\left(t_{1}\right)$ and $Y_{2}=Y\left(t_{2}\right)$. Alternatively, the signal may be received by two distinct mobiles $Y_{1}$ and $Y_{2}$ at the same moment in time. These scenarios may also be reversed to consider the uplink: either the same mobile transmits to the same base station $X$ at moments $t_{1}$ and $t_{2}$ at locations $Y_{1}$ and $Y_{2}$, respectively, or the same base station $X$ simultaneously receives from two distinct mobiles at locations $Y_{1}$ and $Y_{2}$. All four cases can come under the category of autocorrelation and are illustrated in Fig. 1(a).

\section{Cross-Correlation}

This is the central object of our study. Cross-correlation, or site-to-site correlation [73], considers two transmitting base stations $Y_{1}$ and $Y_{2}$ that transmit to a common mobile receiver $X$. Alternatively, cross-correlation can consider a mobile transmitter $X$ whose signal is picked up by two base stations $Y_{1}$ and $Y_{2}$. These are illustrated in Fig. 1(b).

Now, observing the previous section on autocorrelation and the systematic nomenclature we gave to nodes ( $X$ a common node, and $Y_{1}$ and $Y_{2}$ nodes on two separate links), we may abstract from the nature of these nodes and the link direction and conclude that autocorrelation and cross-correlation are very similar in the mathematical sense and can be studied in the same manner. Furthermore, we see in [46] and [74] how an autocorrelation model can also be used to model crosscorrelation. We therefore assume that $X$ is the common node to all paths, which we locate at the origin for simplicity, and all links are between $X$ and the points $Y_{i}$.

\section{Generalised Correlation}

If we allow total freedom for the positions of the two paths, then the correlation model becomes more complex; in fact, it becomes a function of up to eight free variables (four free positions on a 2-D plane).
We will not study this type of correlation for two reasons: first, because of its increased complexity; and second, because the methods given in the literature begin with an explicit construction of the shadowing realisations, and hence, it is not necessary to study their feasibility. They are always feasible. Contrast this with the correlation models studied here. In Section VIII-A, it is necessary to factorise a matrix, which is not always possible, and thus, the method is implicit, and feasibility must be studied.

Such methods rely on generating shadowing maps [42] in some way. Three algorithms for doing so are the sum-ofsinusoids (SoS) algorithm [67], the network shadowing (NeSh) method [2], [3], [44], [68], and the over-obstacle multiple-edge diffraction model [75].

\section{E. Time Correlation}

Time correlation is different in that it only considers one path $\overrightarrow{X Y}$ but looks at the correlation between shadowing at various moments in time. Shadowing can thus be represented as a random process $S(t)$ [8], [64]. The feasibility of the model is a simple problem here because the problem simply evolves in one dimension, i.e., time. The correlation model is then specified by the autocorrelation function of $S(t)$, and the model will be $p s d$ if and only if the autocorrelation function is $p s d$. We are not aware of any measurements of correlation in time only, and it is not evident if shadowing significantly changes over a fixed path.

It should be understood that shadowing evolution in time should (like in space) have fast fading removed through time averaging of some duration.

\section{F. Uplink-Downlink Correlation}

Consider the path $\overrightarrow{X Y}$ and then its return path $\overrightarrow{Y X}$. By channel symmetry, one might conclude that the shadowing experienced in both directions is identical, which would correspond to a correlation coefficient of $\rho_{\mathrm{ud}}=1$. In practice, measurements indicate a high degree of correlation: $\rho_{\mathrm{ud}} \geq 0.66$ [35]. From these measurements, Kim and Han [76] assumed $\rho_{\text {ud }}=0.7$. Furthermore, Kotz et al. [50] demonstrated asymmetry but positive correlation in link connectivity, which can be interpreted as unequal but correlated shadowing in each direction.

\section{Shadowing Model Description}

Autocorrelation and cross-correlation models can generically be described as follows: consider $N$ points $Y_{1}, \ldots, Y_{N}$ located on a plane at positions $\vec{r}_{1}, \ldots \vec{r}_{N}$ with $\vec{r}_{i} \in \mathbb{R}^{2} \backslash\{0\}$. We will write $r_{i}=\left\|\vec{r}_{i}\right\|$. We assume, without loss of generality, that the common point $X$ is located at the origin, and thus, $\vec{r}_{i}=\overrightarrow{X Y}_{i}$. Consider $S_{i}$ as the logarithm of the power attenuation due to shadowing on each path $\vec{r}_{i}$ (see Fig. 2). We have $\mathbb{E}\left\{S_{i}\right\}=$ 0 . We will, for the moment, not commit to any particular shadowing distribution. We only require the condition that $\operatorname{VAR}\left\{S_{i}\right\}<\infty$. 


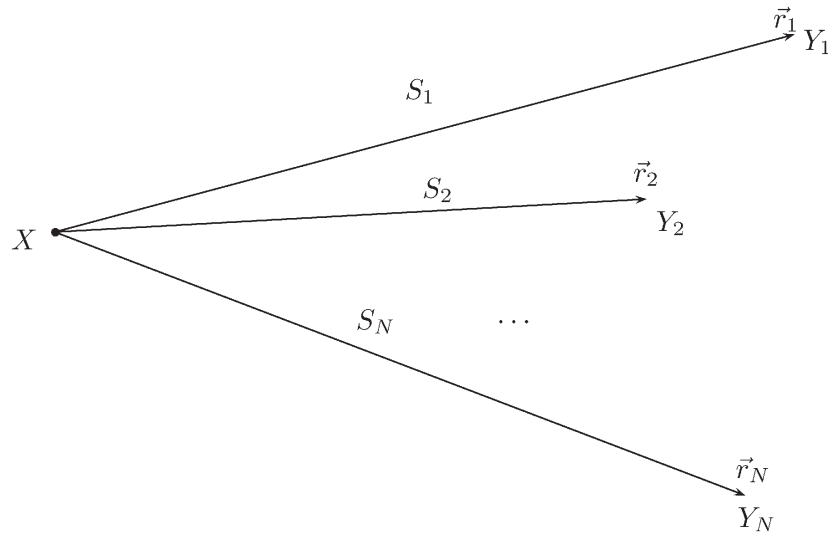

Fig. 2. Generic geometry of shadowing autocorrelation and cross-correlation.

The shadowing log-variance can most generally be characterised as

$$
\sigma_{i}^{2}=\operatorname{VAR}\left\{S_{i}\right\}=\sigma_{\mathrm{s}}^{2}\left(\overrightarrow{r_{i}}\right)
$$

although in an isotropic medium, it will simply be a function of distance: $\mathbb{V A R}\left\{S_{i}\right\}=\sigma_{\mathrm{s}}^{2}\left(r_{i}\right)$. Usually, shadowing log-variance is considered as constant after some distance [1].

The pairwise correlation coefficients necessarily exist (by the Cauchy-Schwarz inequality) and can be defined as

$$
\begin{aligned}
\rho_{i, j} & =\mathbb{E}\left\{S_{i} S_{j}\right\} / \sigma_{i} \sigma_{j}=h\left(\vec{r}_{i}, \vec{r}_{j}\right), \quad i \neq j \\
\rho_{i, i} & =1 .
\end{aligned}
$$

We then have the following properties.

1) $-1 \leq h\left(\vec{r}_{i}, \vec{r}_{j}\right) \leq 1$.

2) $h\left(\vec{r}_{i}, \vec{r}_{j}\right)=h\left(\vec{r}_{j}, \vec{r}_{i}\right)$.

A shadowing model that includes pairwise correlation can most generally be defined by the pair of functions $\left(\sigma_{\mathrm{s}}^{2}(\vec{r}), h\left(\vec{r}_{1}, \vec{r}_{2}\right)\right)$.

Now, the correlation matrix of $\mathbf{S}=\left[S_{1}, \ldots, S_{N}\right]$ is

$$
\mathbf{K}=\left[\begin{array}{cccc}
\sigma_{1}^{2} & \sigma_{1} \sigma_{2} \rho_{1,2} & \cdots & \sigma_{1} \sigma_{N} \rho_{1, N} \\
\sigma_{1} \sigma_{2} \rho_{1,2} & \sigma_{2}^{2} & \cdots & \sigma_{2} \sigma_{N} \rho_{2, N} \\
\vdots & \vdots & \ddots & \vdots \\
\sigma_{1} \sigma_{N} \rho_{1, N} & \sigma_{2} \sigma_{N} \rho_{2, N} & \cdots & \sigma_{N}^{2}
\end{array}\right]
$$

\section{Positive Semidefiniteness of CORRELATION MODElS}

From Property 4, for $\mathbf{K}$ to be a valid covariance matrix of $\mathbf{S}$, it is necessary (but not always sufficient, as seen in Section VIII) that $\mathbf{K}$ be $p s d$ ( $\mathbf{K}$ is already symmetric by construction).

Definition 6: We say that a shadowing model $\left(\sigma_{\mathrm{s}}^{2}(\vec{r})\right.$, $\left.h\left(\vec{r}_{1}, \vec{r}_{2}\right)\right)$ is psd if $\forall N, \forall\left[\vec{r}_{1}, \ldots, \vec{r}_{N}\right] \in\left(\mathbb{R}^{2} \backslash\{0\}\right)^{N}$, the correlation matrix $\mathbf{K}$ is always $p s d$.

\section{A. Models With Variable Shadowing Variance}

Theorem 1: If a model $\left(1, h\left(\vec{r}_{1}, \vec{r}_{2}\right)\right)$ with constant log-variance 1 is $p s d$, then for any $\sigma_{\mathrm{s}}^{2}(\vec{r})$, the model $\left(\sigma_{\mathrm{s}}^{2}(\vec{r}), h\left(\vec{r}_{1}, \vec{r}_{2}\right)\right)$ is also psd. Conversely, if the model $\left(\sigma_{\mathrm{s}}^{2}(\vec{r}), h\left(\vec{r}_{1}, \vec{r}_{2}\right)\right)$ is $p s d$ and $\sigma_{\mathrm{s}}^{2}(\vec{r})>0 \forall \vec{r} \in \mathbb{R}^{2} \backslash\{0\}$, then the model $\left(1, h\left(\vec{r}_{1}, \vec{r}_{2}\right)\right)$ with constant log-variance 1 is $p s d$.

Proof: Consider $N$ shadowing paths $\vec{r}_{i} \in \mathbb{R}^{2} \backslash\{0\}$ and a shadowing model $\left(\sigma_{\mathrm{s}}^{2}(\vec{r}), h\left(\vec{r}_{1}, \vec{r}_{2}\right)\right)$, where $\left(1, h\left(\vec{r}_{1}, \vec{r}_{2}\right)\right)$ is a psd shadowing model. We call $\mathbf{H}$ the $N \times N$ matrix with entries $h\left(\vec{r}_{i}, \vec{r}_{j}\right)$ and $\mathbf{s}$ the column vector with entries $\sigma_{\mathrm{s}}\left(\vec{r}_{i}\right)$. Then, the correlation matrix of $\mathbf{S}$ can be written as

$$
\mathbf{K}=\left(\mathbf{s s}^{\mathrm{T}}\right) \circ \mathbf{H} .
$$

Now, the matrix $\mathbf{s s}^{\mathrm{T}}$ is $p s d$, as can be seen from Definition 2: $\mathbf{x}^{\mathrm{T}}\left(\mathbf{s s}^{\mathrm{T}}\right) \mathbf{x}=\left(\mathbf{x}^{\mathrm{T}} \mathbf{s}\right)^{2} \geq 0 \forall \mathbf{x} \in \mathbb{R}^{N}$. In addition, since $\left(1, h\left(\vec{r}_{1}, \vec{r}_{2}\right)\right)$ is $p s d$, it follows that $\mathbf{H}$ is $p s d$. Applying the Schur product theorem, we find that $\mathbf{K}$ is always $p s d$, which implies that the model $\left(\sigma_{\mathrm{s}}^{2}(\vec{r}), h\left(\vec{r}_{1}, \vec{r}_{2}\right)\right)$ is $p s d$.

To prove the converse, we write

$$
\mathbf{H}=\left(\mathbf{z z}^{\mathrm{T}}\right) \circ \mathbf{K}
$$

where $\mathbf{z}$ is the column vector with entries $\sigma_{\mathrm{s}}^{-1}\left(\vec{r}_{i}\right)$, and the proof is analogous. Of course, here, the additional requirement that $\sigma_{\mathrm{s}}(\vec{r}) \neq 0 \forall \vec{r} \in \mathbb{R}^{2} \backslash\{0\}$ is required.

Thus, to study the positive semidefiniteness of a shadowing model, it is sufficient to study the correlation function $h\left(\vec{r}_{1}, \vec{r}_{2}\right)$ in isolation, which simplifies the problem. We will therefore say that $h$ is $p s d$ if $(1, h)$ is $p s d$.

\section{B. Methods for Proving Positive Semidefiniteness}

While we do not have a general criterion for proving that some given $h$ is psd or not, the following approaches will nevertheless help us analyze most particular cases.

1) If there exists an explicit constructive algorithm for generating data according to $h$, then $h$ is necessarily $p s d$, since the resulting covariance matrix will always be $p s d$ (see Property 4).

2) If there exists at least one choice of $\left[\vec{r}_{1}, \ldots, \vec{r}_{N}\right]$ for which $\mathbf{K}$ is not $p s d$, then $h$ is not $p s d$. For this test, we need $N \geq 3$, since every correlation matrix of size 2 is $p s d$, as seen from this decomposition:

$$
\begin{aligned}
{\left[\begin{array}{ll}
1 & \rho \\
\rho & 1
\end{array}\right] } & =\left[\begin{array}{ll}
u & v \\
v & u
\end{array}\right]^{2}, \quad-1 \leq \rho \leq 1 \\
u & =\frac{1}{2} \sqrt{1+\rho}+\frac{1}{2} \sqrt{1-\rho} \\
v & =\frac{1}{2} \sqrt{1+\rho}-\frac{1}{2} \sqrt{1-\rho} .
\end{aligned}
$$

However, $N=3$ may not be enough [57].

3) Several theorems can also be used to prove that some $h$ is $p s d$, as seen in the next section. 


\section{One-Parameter and Separable Correlation}

Proving that isotropic models (functions of $d=\left\|\vec{r}_{1}-\vec{r}_{2}\right\|$ only, with $\vec{r}_{i} \in \mathbb{R}^{2}$ ) are psd is not trivial. Indeed, while for all psd models $h(d)$ the function $h(x)$ on $\mathbb{R}^{+}$is positive definite, the converse is not true, and tighter conditions are needed [66, p. 361]. The following two theorems are examples of tests that can be applied to verify that $h(d)$ is $p s d$.

Theorem 2: A one-parameter correlation model $h(d)$ with $d=\left\|\vec{r}_{1}-\vec{r}_{2}\right\|$ with $\vec{r}_{i} \in \mathbb{R}^{2}$ is $p s d$ if $^{2}$ the integral

$$
f(\omega)=\int_{0}^{\infty} h(x) J_{0}(\omega x) x d x
$$

exists and is nonnegative $\forall \omega \geq 0$ [66, p. 357]. $J_{0}(x)$ is the Bessel function of the first kind of order 0 .

Theorem 3: A one-parameter correlation model $h(d)$ with $d=\left\|\vec{r}_{1}-\vec{r}_{2}\right\|$ with $\vec{r}_{i} \in \mathbb{R}^{2}$ is $p s d$ if we have the following ${ }^{2}$ :

1) the function $h(x)$ with $x \in \mathbb{R}^{+}$is positive definite;

2) the Fourier transform $f(\omega)$ of $h(|x|)$ is nonincreasing on $\omega \in(0, \infty)$.

The converse is not true [66, p. 361].

Many other useful properties of isotropic correlation models are found in [66, Ch. 22].

Theorem 4: A one-parameter correlation model $h(\theta)$ with $\theta=\left|\angle \vec{r}_{1}-\angle \vec{r}_{2}+2 k \pi\right|, k \in \mathbb{Z}$, such that $\theta \in\left[0^{\circ}, 180^{\circ}\right]$ is $p s d$ if it may be written as

$$
h(\theta)=\sum_{n=0}^{\infty} a_{n} \cos (n \theta)
$$

for some nonnegative and bounded sequence $a_{0}, a_{1}, \ldots$ with $0<\sum_{n=0}^{\infty} a_{n}<\infty$.

Proofs are given in [55] and [56].

Theorem 5: A one-parameter correlation model $h(|x|)$ with $|x|=\left|g\left(\vec{r}_{1}\right)-g\left(\vec{r}_{2}\right)\right|$ for some function $g: \mathbb{R}^{2} \backslash\{0\} \mapsto \mathbb{R}$ is psd if it may be written as

$$
h(x)=\int_{0}^{\infty} \cos (2 \pi x \omega) f(\omega) d \omega
$$

for some nonnegative and finite-area $f(\omega)$ on $0<\omega<\infty$.

Proof: From Bochner's theorem, $h(x)$ in (11) is a psd function. From Definition 5, it follows that for any $N$ the matrix $\mathbf{H}_{N \times N}$ with entries $\rho_{i, j}=h\left(\left|t_{i}-t_{j}\right|\right), t_{i}=g\left(\vec{r}_{i}\right)$ is $p s d$.

Theorem 6: If a correlation model $h$ may be written as

$$
h\left(\vec{r}_{1}, \vec{r}_{2}\right)=h_{1}\left(\vec{r}_{1}, \vec{r}_{2}\right) h_{2}\left(\vec{r}_{1}, \vec{r}_{2}\right)
$$

with $h_{1}$ and $h_{2}$ both $p s d$, then $h$ is also $p s d$.

Proof: Let $\mathbf{H}, \mathbf{H}_{1}$, and $\mathbf{H}_{\mathbf{2}}$ be the $N \times N$ matrices with entries $h\left(\vec{r}_{i}, \vec{r}_{j}\right), h_{1}\left(\vec{r}_{i}, \vec{r}_{j}\right)$, and $h_{2}\left(\vec{r}_{i}, \vec{r}_{j}\right)$, respectively. We may then write $\mathbf{H}=\mathbf{H}_{\mathbf{1}} \circ \mathbf{H}_{\mathbf{2}}$. Now, since $h_{1}$ and $h_{2}$ are $p s d$, it follows that $\mathbf{H}_{1}$ and $\mathbf{H}_{2}$ are both $p s d$. Applying Schur's product theorem, we have that $\mathbf{H}$ is also psd, which implies that $h$ is psd.

\footnotetext{
${ }^{2}$ These two theorems apply for shadowing on $\mathbb{R}^{2}$ and take a more restricted form on $\mathbb{R}^{3}[66]$.
}

A similar argument was given in [56] but for positive definite instead of $p s d$ matrices.

\section{Incorporating Time and Uplink-Downlink Correlation}

As shown in Section II-E, shadowing may evolve in time in a correlated manner. In addition, as seen in Section II-F, shadowing may be different on the same propagation path in both directions, although these are usually highly correlated. We will show that, given a positive definite temporal autocorrelation $R_{S}(\tau)$ and a psd spatial correlation model $h$, and assuming correlation separability between the spatial, time, and uplink-downlink dimensions, the resulting combined correlation matrix is always $p s d$. Combining cross-correlation with temporal autocorrelation was described in [8].

Consider a common node $X$ and $N$ nodes $Y_{1}, \ldots, Y_{N}$. Let $S_{i}(t)$ be the shadowing on path $\overrightarrow{X Y}_{i}$ at time instant $t$ with variance $\sigma_{i}^{2}=\sigma_{\mathrm{s}}^{2}\left(\vec{r}_{i}\right)$ and $\tilde{S}_{i}$ the shadowing on the return path $\overrightarrow{Y_{i} X}$ with variance $\tilde{\sigma}_{i}^{2}=\tilde{\sigma}_{\mathrm{s}}^{2}\left(\vec{r}_{i}\right)$.

Consider the spatial correlation function $h$ such that $\rho_{i, j}=$ $h\left(\vec{r}_{i}, \vec{r}_{j}\right), i \neq j$, and $\rho_{i, i}=1$, i.e.,

$$
\begin{aligned}
\mathbb{E}\left\{S_{i}(t) S_{j}(t)\right\} & =\sigma_{i} \sigma_{j} \rho_{i, j} \quad \forall t \\
\mathbb{E}\left\{\tilde{S}_{i}(t) \tilde{S}_{j}(t)\right\} & =\tilde{\sigma}_{i} \tilde{\sigma}_{j} \rho_{i, j} \quad \forall t .
\end{aligned}
$$

We will assume that the correlation between the two directions of the same path is constant, as in [76], i.e.,

$$
\mathbb{E}\left\{S_{i}(t) \tilde{S}_{i}(t)\right\} / \sigma_{i} \tilde{\sigma}_{i}=\rho_{\mathrm{ud}} \quad \forall i \forall t
$$

Consider also the normalised temporal autocorrelation function $\zeta(\tau)$, i.e.,

$\frac{\mathbb{E}\left\{S_{i}(t) S_{i}(t+\tau)\right\}}{\sigma_{i}^{2}}=\frac{\mathbb{E}\left\{\tilde{S}_{i}(t) \tilde{S}_{i}(t+\tau)\right\}}{\tilde{\sigma}_{i}^{2}}=\zeta(\tau) \quad \forall i \forall t$

where $\zeta(\tau)$ is positive definite, and $\zeta(0)=1$.

We will further assume separability of cross-correlation and uplink-downlink correlation so that the correlation terms can be found as follows:

$$
\begin{aligned}
& \frac{\mathbb{E}\left\{S_{i}\left(t_{1}\right) S_{j}\left(t_{2}\right)\right\}}{\sigma_{i} \sigma_{j}}=\frac{\mathbb{E}\left\{\tilde{S}_{i}\left(t_{1}\right) \tilde{S}_{j}\left(t_{2}\right)\right\}}{\tilde{\sigma}_{i} \tilde{\sigma}_{j}}=\rho_{i, j} \zeta\left(t_{2}-t_{1}\right) \\
& \mathbb{E}\left\{S_{i}\left(t_{1}\right) \tilde{S}_{j}\left(t_{2}\right)\right\}=\sigma_{i} \tilde{\sigma}_{j} \rho_{\mathrm{ud}} \rho_{i, j} \zeta\left(t_{2}-t_{1}\right) .
\end{aligned}
$$

From this equation, we see that separability implies that the uplink and downlink cross-correlation models must be the same, i.e., $h=\tilde{h}$.

Consider $M$ time instances $t_{1}, \ldots, t_{M}$. Then, the correlation matrix of $S_{1}\left(t_{1}\right), \tilde{S}_{1}\left(t_{1}\right), \ldots, S_{N}\left(t_{1}\right), \tilde{S}_{N}\left(t_{1}\right), \ldots, S_{1}\left(t_{M}\right)$, $\tilde{S}_{1}\left(t_{M}\right), \ldots, S_{N}\left(t_{M}\right), \tilde{S}_{N}\left(t_{M}\right)$ is

$\begin{aligned} & \overline{\mathbf{K}}_{2 N M \times 2 N M}= {\left[\zeta\left(t_{j}-t_{i}\right)\right]_{M \times M} } \\ & \otimes\left(\left(\breve{\mathbf{S}}^{\mathrm{T}}\right) \circ\left(\left[\rho_{i, j}\right]_{N \times N} \otimes\left[\begin{array}{cc}1 & \rho_{\mathrm{ud}} \\ \rho_{\mathrm{ud}} & 1\end{array}\right]\right)\right)\end{aligned}$

where $\breve{\mathbf{s}}$ is the column vector with entries $\sigma_{1}, \tilde{\sigma}_{1}, \ldots, \sigma_{N}, \tilde{\sigma}_{N}$. 
Theorem 7: Given a psd correlation function $h$, uplink and downlink shadowing variances $\sigma_{\mathrm{s}}^{2}(\vec{r})$ and $\tilde{\sigma}_{\mathrm{s}}^{2}(\vec{r})$, an uplink-downlink correlation coefficient $-1 \leq \rho_{\mathrm{ud}} \leq 1$, and a normalised shadowing positive definite time autocorrelation function $\zeta(\tau)$ with $\zeta(0)=1$, and assuming that the correlation is separable, as in (16), we can conclude that the composite correlation model is $p s d$.

Proof: Consider (17). The matrix $\breve{\mathbf{S}}^{\mathrm{T}}$ is always $p s d$, as seen in the proof of Theorem 1. The matrix $\left[\zeta\left(t_{j}-t_{i}\right)\right]_{M \times M}$ is psd because $\zeta(\tau)$ is positive definite, and the matrix $\left[\rho_{i, j}\right]_{N \times N}$ is $p s d$ because $h$ is $p s d$. Finally, all $2 \times 2$ correlation matrices are $p s d$, as seen in (8). Both the Hadamard and the Kronecker products are $p s d$ if both their factors are $p s d$, as seen in Properties 1 and 2. It follows that $\overline{\mathbf{K}}$ is $p s d$.

Simply speaking, one can incorporate (separable) directional and time correlation without upsetting the positive semidefiniteness of the correlation model. Other dimensions, notably frequency [49], may also be similarly incorporated.

One can note that the Kronecker product has similarly been used in [77] in a multiantenna context and in [45] for correlation between shadowing, delay spread, and angle spread, all under the assumptions of separability, which is natural when no further information is available [45].

\section{Estimating THE CORRELATION FUnCtion FROM MEASURED DATA}

To better understand why the estimated models may be $p s d$ or not, it is worthwhile to examine some aspects of how these models are constructed from measured data.

\section{A. Autocorrelation as Mixed Time-Space Measurements}

Autocorrelation is measured [24]-[26], [28], [32], [36]-[39] by taking a single moving mobile $Y$ with velocity $v$ in uniform rectilinear motion, measuring the power loss between $Y$ and a base station $X$, correcting for path loss and small-scale fading, and considering the shadowing $S$ along the path $\overrightarrow{X Y}$ (or $\overrightarrow{Y X}$ ) as a process of time $S(t)$ (equivalently, of space $S(d)$, with $d=$ $v t$ [78], [79]). The underlying assumption is that shadowing over a fixed link does not vary with time (or perhaps varies little or very slowly compared with variation when in motion). More specifically, consider the shadowing $S(\vec{r}, t)$ as would be experienced by a virtual mobile $\tilde{Y}$ at time $t$ and location $\vec{r}$. Now, let the real mobile $Y$ begin at location $\vec{r}_{0}$ at time $t=0$ and move in uniform rectilinear motion with velocity $\vec{v}$. Thus, its location at time $t$ is $\vec{r}=\vec{r}_{0}+t \vec{v}$. The observed shadowing process at $Y$ is thus $S(t)=S\left(\vec{r}_{0}+t \vec{v}, t\right)$, or equivalently, $S(d)=S\left(\vec{r}_{0}+\right.$ $d \vec{v} / v, d / v)$. Clearly, the observed process varies in both time and space, and assuming that $S(\vec{r}, t)$ varies in $t$ when $\vec{r}$ is fixed (see Section II-E), these measurements do not directly translate into a correlation model. For example, a different model $S(d)$ might be extracted if the velocity $v$ is changed. This is generally true for other motion trajectories.

An additional issue about autocorrelation models is the levelcrossing rate, which is only properly defined for some models. This topic is addressed in [61], [62], and [79]-[81], and we do not further explore it here.

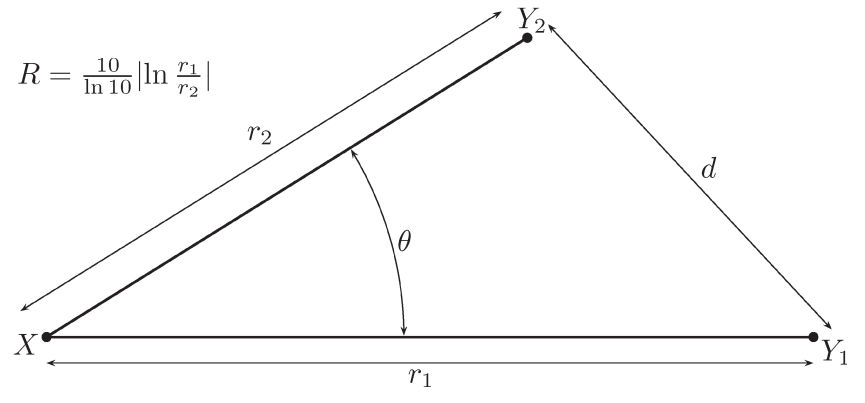

Fig. 3. Pair of autocorrelated or cross-correlated paths with the most relevant dimensional variables: $d, \theta, R$.

\section{B. Cross-Correlation as Spatial-Only Correlation}

Several field measurements have been performed [18], [24], [28], [29], [33], [34], [36]-[38] to gather data about the true physical correlation model, which we call $\bar{h}$. Field measurements produce a set of data points, from which an estimate of the cross-correlation function, which we call $\hat{h}$, is obtained by fitting the data with plausible-looking simple mathematical functions. It must be understood that $\bar{h}$ is always a $p s d$ model by its very mathematical definition in (4). It was argued in [57] that a particularly convenient $p s d$ model $\hat{h}$ may not accurately reflect the true correlation. This is true. However, because $\bar{h}$ must be $p s d$, it is likely that there exists some $p s d$ model $\hat{h}$ that is close to $\bar{h}$. Therefore, we argue that it is always best and possible to choose a psd model $\hat{h}$ that is also close to $\bar{h}$.

In general, $\bar{h}\left(\vec{r}_{1}, \vec{r}_{2}\right)$ is a function of four variables. However, estimating a four-variable function accurately requires many more points than can realistically be obtained from costly and cumbersome field measurements. Additionally, every point of the function $\bar{h}$ is itself estimated as an expectation (4) and requires several data points. Two consequences follow.

1) The measurement data are usually grouped by collapsing them from four variables into a single variable.

2) On that one variable, the curve fitting is still relatively crude [26], [28], [31], [32], [34], [39].

These two approximation steps further distance $\hat{h}$ from $\bar{h}$ and may cause the model $\hat{h}$ to be non-psd. Nevertheless, for practical reasons, it is simplest to measure correlation along one dimension only [66, pp. 358-359].

\section{Collapsing Correlation Onto One Dimension}

The measurements can be grouped along one variable in several ways. In such cases, $\hat{h}$ is expressed as a function of a single free variable. The most common forms are the following:

1) absolute distance (between $Y_{1}$ and $Y_{2}$ ) [24]-[26], [28], [31], [32], [36]-[39]: $d=\left\|\vec{r}_{1}-\vec{r}_{2}\right\|$;

2) angle (not oriented) of arrival separation [24], [28], [33], [34], [36]-[38], [56]: $\theta=\left|\angle \vec{r}_{1}-\angle \vec{r}_{2}\right| \in\left[0^{\circ}, 180^{\circ}\right]$;

3) arrival distance ratio (in decibels) [33], [37], [56], [72]: $R=\left|10 \log _{10} r_{1} / r_{2}\right|=(10 / \ln 10)\left|\ln r_{1}-\ln r_{2}\right|$.

These quantities are illustrated in Fig. 3.

For correlation functions expressed in $d$, one may also estimate and fit the power spectral density (which is the Fourier transform of the autocorrelation function in differences of $d$ ) of the measured data to that of a particular model [28], [32]. 
TABLE I

Summary of Existing Shadowing CORRElation Models and Their Properties

\begin{tabular}{|c|c|c|c|c|c|c|}
\hline Model Name & Equation & Tunable Parameters & References & Dimensions & psd & Physical Properties $^{b}$ Violated \\
\hline Constant & (18) & $0<\rho<1$ & many & none & Yes & $1,5,7$ \\
\hline $\operatorname{Exp}(d)$ & (19) & $d_{0}>0$ & {$[25],[26], \ldots$} & $d$ & Yes & 7 \\
\hline $\operatorname{Exp}+\operatorname{Exp}(d)$ & (20) & $\begin{array}{l}0 \leq a \leq 1 \\
0<d_{1}, 0<d_{2}\end{array}$ & {$[28],[32],[90]$} & $d$ & Yes & 7 \\
\hline Gaussian $(d)$ & (21) & $\bar{d}>0$ & [79] & $d$ & Yes & 7 \\
\hline $\operatorname{Exp} * \operatorname{Gaussian}(d)$ & $(22)$ & $d_{0}>0, \bar{d}>0$ & [81] & $d$ & Yes & 7 \\
\hline \multirow[t]{2}{*}{$\operatorname{Exp}\left(d^{\nu}\right)$} & (23) & $d_{0}>0,0<\nu \leq 2$ & [91] & $d$ & Yes & 7 \\
\hline & & $d_{0}>0, \nu>2$ & & & No & 7 \\
\hline $\operatorname{Cos}(\theta)$ & (24) & $\begin{array}{l}A \geq 0, B \geq 0 \\
A+B \leq 1\end{array}$ & [15], [55], [84], [92] & $\theta$ & Yes & $(1)^{c},(2)^{c}, 4,(5)^{c}$ \\
\hline Piecewise $(\theta)$ & (25) & none & {$[34]$} & $\theta$ & No & 1,6 \\
\hline $\operatorname{Stepwise}(\theta)$ & (26) & none & {$[33$, Table 1] } & $\theta$ & No & $1,5,6$ \\
\hline Triangular $(\theta)$ & (27) & $\begin{array}{l}0 \leq b<a \leq 1 \\
0^{\circ}<\theta_{0}<180^{\circ}\end{array}$ & {$[72]$} & $\theta$ & Yes & $(1)^{c},(2)^{c},(5)^{c}$ \\
\hline $\operatorname{Exp}(\theta)$ & $(28)$ & $\alpha>0$ & [4], [41], [56], [93] & $\theta$ & Yes & $2,(5)^{c}$ \\
\hline$" 1.0 / 0.0 \mathrm{RX} "$ & $\begin{array}{l}(29),(30), \\
(31)\end{array}$ & $R_{0}>0$ & [72] & $\theta, R$ & No & 6 \\
\hline \multirow[t]{2}{*}{ Triangular $(\theta) \times$ Triangular $(R)$} & $\begin{array}{l}(29),(27), \\
(31)\end{array}$ & $\begin{array}{l}0 \leq b<a \leq 1,0^{\circ}< \\
\theta_{0}<180^{\circ}, R_{0}>0\end{array}$ & modified [72] & $\theta, R$ & Yes & $(1)^{c},(5)^{c}$ \\
\hline & & $b=0, a=1$ & & & Yes & none \\
\hline $\operatorname{Exp}(d) \times\left(\theta \leq 90^{\circ}\right)$ & $(32)$ & $d_{0}>0$ & [46] & $d, \theta$ & No & 6 \\
\hline $\operatorname{Exp}(d) \times \cos ^{+}(\theta)$ & (33) & $d_{0}>0$ & [94] & $d, \theta$ & No & none \\
\hline $\operatorname{Exp}(d) \times \cos (\theta)$ & (34) & $d_{0}>0$ & modified [94] & $d, \theta$ & Yes & 4 \\
\hline Saunders' & (35) & $d_{0}>0, \gamma>0$ & [73], [96] & $\begin{array}{l}\left(\theta, r_{1}, r_{2}\right)^{a} \\
r_{i}>d_{0} / 2\end{array}$ & No & $2,(5)^{c}$ \\
\hline Modified Saunders' & (36) & $d_{0}>0, \gamma>0$ & {$[1]$} & $\begin{array}{l}\left(\theta, r_{1}, r_{2}\right)^{a}, \\
r_{2}, \ldots, r_{N}> \\
d_{0} / 2\end{array}$ & No & $2,3,(5)^{c}, 6$ \\
\hline $\operatorname{Stepwise}(\theta, R)$ & $(37)$ & none & [33, Table 2] & $(\theta, R)^{a}$ & No & $1,5,6$ \\
\hline $\operatorname{Piecewise}(\theta)-\operatorname{Triangular}^{\alpha}(R)$ & $(38)$ & $\begin{array}{l}R_{0}>0, \alpha \geq 0, a> \\
0, b>0, a+b \leq 0.22\end{array}$ & {$[22]$} & $(\theta, R)^{a}$ & No & $(1)^{c},(5)^{c}, 6$ \\
\hline$" 1.0 / 0.4$ RX" & $(39)$ & $R_{0}>0$ & {$[72]$} & $(\theta, R)^{a}$ & No & 5,6 \\
\hline
\end{tabular}

${ }^{a}$ Non-separable

${ }^{b}$ As described in Section VII

${ }^{c}$ Physical property met only for some parameter choices

\section{SPeCific CorRelation Models AND THEIR Positive SEMIDEFINITENESS}

We have made a wide (if not exhaustive) investigation of the correlation models $h$ used in the literature. All existing models imply jointly lognormal shadowing (which is by no means a requirement of our analysis). Most models assume a constant shadowing variance $\sigma_{\mathrm{s}}^{2}(\vec{r})=\sigma_{\mathrm{s}}^{2}$ but not all [1], [39]. As we have shown in Theorem 1, the positive semidefiniteness of a shadowing model is separate from the shadowing spread function. As such, we may safely study the existing correlation models while looking forward to more complex point-to-point shadowing spread models in the future.

The various existing models and their properties are summarised in Table I. In particular, we show which models are $p s d$. While [45] stated that "most" models are non-psd, we find that actually a slight majority of the models are in fact $p s d$.

It should be understood that these models are not necessarily mutually exclusive when they are expressed in differ- ent $(d, R, \theta)$ domains, since this implies a different reduction (integration) of the original 4-D model $\bar{h}$ onto one or two dimensions, possibly representing different projections of the same $\bar{h}$ onto those dimensions.

\section{A. Constant Model}

The simplistic model that assigns $\rho_{i, j}=\rho, i \neq j$

$$
h\left(\vec{r}_{1}, \vec{r}_{2}\right)=\rho
$$

with $0<\rho<1$, is sometimes used [5], [9]-[11], [20], [21], [29], [42], [45], [47], [53], [61], [63], [76], [82], [83] $(\rho=$ 0.5 [7], [23], [59], [60]) when more information is lacking. However, [7] argued that this may be a too-simplistic model, comparing simulations that use constant versus nonconstant models. On the other hand, we have shown [13] that for a high number of highly correlated lognormal-shadowed interferers, the total interference power may be well approximated with 
the knowledge of the average correlation coefficient only. (It is important to actually estimate this average. This was not done in [7].)

The model is claimed $p s d$ for $\rho=0.5$ in [45]. It does, in fact, give a $p s d \mathbf{H}$ for $\rho \geq-1 /(N-1)$ [83], and therefore, the model is $p s d$ according to Definition 6 for $0<\rho<1$ (as well as $\rho=0,1$ ) but would be non-psd for $-1 \leq \rho<0$. It can quickly be simulated [42], [83] in the case of jointly lognormal correlated shadowing.

\section{B. Absolute Distance-Only Models}

All the autocorrelation models are expressed as a function of $d$ (equivalently, of time $t$, given a constant velocity $v$ ).

1) Exponential Model: The most common autocorrelation model is a decaying exponential of distance [6], [8], [25], [26], [31], [36], [38]-[40], [42], [45], [48], [49], [51], [52], [56], [62], [70], [73], [74], [78], [80], [84]-[88]. This model is often attributed to Gudmundson [26], although the less-cited work of Marsan et al. [25] also proposes this model, and measurements by Graziano [24, Figs. 5 and 7] suggest it. This model has also been interpreted as a cross-correlation model [74]. It may be expressed as

$$
h(d)=e^{-d / d_{0}}
$$

where $d_{0}>0$ is the tunable parameter called the decorrelation distance (sometimes defined for $50 \%$ correlation rather than $1 / e$ ). Literature surveys have been made [85], [86] of the values that $d_{0}$ might take in field measurements. While this model can be understood as being based on a first-order autoregressive (AR(1)), i.e., first-order Markov process [45], its positive semidefiniteness is not thereby evident, since, on a nonlinear trajectory, nonsuccessive points may have different correlation coefficients than simply those constructed by an AR(1) filter. The fact that $e^{-x / d_{0}}$ is a $p s d$ function on $\mathbb{R}^{+}$is a necessary but not sufficient condition [66, p. 361] (the same applies for any model depending on $d$ ). Nevertheless, this model is proved to be $p s d$ using Theorem 2 in [66, p. 362] and similarly in [56].

For equally spaced ordered points $Y_{1}, \ldots, Y_{N}$ on a straight line with separation $d_{\text {sep }}$, we have the correlation coefficients equal to $\rho_{i, j}=\rho^{|i-j|}$, where $\rho=e^{-d_{\mathrm{sep}} / d_{0}}$. We then have a correlation matrix as in [89], which is necessarily $p s d$, because the model (19) is $p s d$.

2) More Complex Models: The exponential model has inspired some more complex models, which may be interpreted as autocorrelation or cross-correlation models.

In [28], [32], and [90], the sum of two independent exponential processes is used, which leads to the following correlation model:

$$
h(d)=a e^{-d / d_{1}}+(1-a) e^{-d / d_{2}}
$$

where $0 \leq a \leq 1,0<d_{1}$, and $0<d_{2}$ are tunable parameters. This model is always $p s d$.

Proof: Applying (20) in (9), we may separate the resulting integral by linearity into two integrals, both of which are nonnegative, as seen for the model (19), and weighed by the nonnegative coefficients $a$ and $1-a$. The resulting integral is thus nonnegative. It follows from Theorem 2 that this model is $p s d$.

In [61] and [79] (and implicitly in [81]), a Gaussian correlation model is used, i.e.,

$$
h(d)=e^{-(d / \bar{d})^{2}}
$$

where $\bar{d}>0$ is the tunable parameter. This model is proved to be $p s d$ using Theorem 2 in [66, p. 364].

In [81], a convolution of an exponential and a Gaussian function is proposed, i.e.,

$$
\begin{aligned}
h(d) & =K e^{-|d| / d_{0}} * e^{-(d / \bar{d})^{2}} \\
& =K \int_{-\infty}^{\infty} e^{-|d-t| / d_{0}-t^{2} / \bar{d}^{2}} d t
\end{aligned}
$$

where $d_{0}>0$ and $\bar{d}>0$ are the tunable parameters, and $K=\exp \left(-\bar{d}^{2} / 4 d_{0}^{2}\right) / \sqrt{\pi} \bar{d}$. This model is $p s d$.

Proof: The Fourier transform of (22) with $x=d$ is

$$
f(\omega)=K \frac{2 / d_{0}}{\omega^{2}+d_{0}^{-2}} \bar{d} \sqrt{\pi} e^{-\frac{1}{4}(\bar{d} \omega)^{2}}
$$

which meets the conditions of Theorem 3 .

In [91], a model is proposed that can be written as

$$
h(d)=e^{-\left(d / d_{0}\right)^{\nu}}
$$

with $d_{0}>0$ and $\nu>0$ as tunable parameters. The positive semidefiniteness of this model is dependent on $\nu$ : for $0<\nu \leq$ 2 , it is proved to be $p s d$ with the aid of Theorem 3 in $[66$, p. 364]. This of course includes models (19) and (21), as well as the choice of $\nu=0.9682$ in [91]. For $\nu>2$, however, the model is in non- $p s d$ [66, p. 137]. As a counterexample, consider three aligned points with equal consecutive spacings of $0.2 d_{0}$, for which we find that the correlation matrix is not psd for $\nu=2.1,2.2,2.5,3,5,50$.

\section{Angle-Only Models}

Some of the first shadowing correlation measurements along $\theta$ were reported in [18] and [24], but no analytical model was extracted. A similar measurement campaign [37] reported a much lower angular correlation, suggesting the need for a more complex model. However, it has been argued [45] that $\theta$ is the most significant parameter in cross-correlation, which justifies using these models as a first approximation.

In [55] and [84], and later in [15] and [92], a cosine model was proposed, i.e.,

$$
h(\theta)=A \cos \theta+B
$$

with two tunable parameters $A \geq 0$ and $B \geq 0, A+B \leq 1$. The model was used and assumed psd in [45]. Typical parameter choices have been $A=0.3, B=0.5$ [17], [19], [21], [62] and $A=0.3, B=0.699(9)$ [15], [92].

The model was proved to be $p s d$ in [6], [55], and [56] using Theorem 4 by setting $a_{0}=A, a_{1}=B$, and all other $a_{n}=0$. 
In [34], a piecewise-linear model was proposed, i.e.,

$$
h(\theta)= \begin{cases}0.78-7 \theta / 1250^{\circ}, & 0^{\circ} \leq \theta<15^{\circ} \\ 0.48-7 \theta / 1250^{\circ}, & 15^{\circ} \leq \theta<60^{\circ} \\ 0, & 60^{\circ} \leq \theta \leq 180^{\circ} .\end{cases}
$$

It was shown in [57] that this model is non-psd. Here is an example: $N=7$ with $\angle \vec{r}_{i}=0,5, \ldots, 30^{\circ}$.

In [33, Tab. I], a lookup table for intervals of $\theta$, which is effectively a piecewise-constant model, was given, i.e.,

$$
h(\theta)= \begin{cases}0.6, & 0^{\circ} \leq \theta<30^{\circ} \\ 0.25, & 30^{\circ}<\theta<60^{\circ} \\ \alpha \geq 0.2, & \theta \geq 90^{\circ} .\end{cases}
$$

This model is undefined for $\theta \in\left\{30^{\circ}\right\} \cup\left[60^{\circ}, 90^{\circ}\right]$. We understand that a value for $\alpha$ may be chosen on [0.2, 0.25]. Regardless of the missing information, the model is non- $p s d$, as shown in the following example: $N=11$ with $\angle \vec{r}_{i}=0,4, \ldots, 40^{\circ}$.

A triangular model in $\theta$ was proposed in [72], i.e.,

$$
h(\theta)= \begin{cases}a-(a-b) \theta / \theta_{0}, & \theta \leq \theta_{0} \\ b, & \theta>\theta_{0}\end{cases}
$$

with $0 \leq b<a \leq 1$, and $0^{\circ}<\theta_{0} \leq 180^{\circ}$ : three tunable parameters. In [72], the model $a=0.8, b=0.4$, and $\theta_{0}=60^{\circ}$ was used to fit the measurements in [24]. The same choice of parameters was proposed for cross-correlation modeling for the 802.16-m standard [87]. In addition, $a=0.8, b=0$, $\theta_{0}=60^{\circ}$ was proposed to fit the measurements in [34], which were previously fitted with (25). In [28], the same model with $a=0.9, b=0$, and $\theta_{0}=180^{\circ}$ was used. This model is always $p s d$ for any choice of parameters.

Proof: Consider

$$
\begin{aligned}
& a_{0}=b+(a-b) \frac{\theta_{0}}{2 \pi} \\
& a_{n}=2(a-b) \frac{1-\cos \theta_{0} n}{\pi \theta_{0} n^{2}} .
\end{aligned}
$$

Using Theorem 4 yields (27).

A decaying exponential angle model has also been proposed [4], [41], [56], [93], i.e.,

$$
h(\theta)=e^{-\alpha \theta}
$$

with $\alpha>0$ as a tunable parameter. This model can be seen as inspired from the exponential $d$ model (19), which it approximates for small $\theta$. This model is $p s d$ [56].

Proof: Consider

$$
\begin{aligned}
& a_{0}=\frac{1-e^{-\alpha \pi}}{\alpha \pi} \\
& a_{n}=\frac{2}{\pi} \frac{\alpha}{n^{2}+\alpha^{2}}\left(1-e^{-\alpha \pi}(-1)^{n}\right) .
\end{aligned}
$$

Using Theorem 4 yields (28).

\section{Separable Models}

Separable models are easily constructed from the multiplication of 1-D models. Theorem 6 shows that if the component 1-D models are $p s d$, then so is the composite separable model.

1) Angle-Distance Ratio: Separable $\theta-R$ models may always be written as

$$
h(\theta, R)=h_{\Theta}(\theta) h_{\mathrm{R}}(R) .
$$

The use of the $\theta$ and $R$ dimensions for shadowing correlation models has been argued in [37], [38], [45], [56], [62], and [72], although separability may be a simplistic assumption [56].

In [72], the model "1.0/0.0 RX" is given as

$$
\begin{aligned}
& h_{\Theta}(\theta)= \begin{cases}1-\theta / 75^{\circ}, & \theta \leq 60^{\circ} \\
0, & \theta \leq 60^{\circ}\end{cases} \\
& h_{\mathrm{R}}(R)=\max \left(0,1-R / R_{0}\right)
\end{aligned}
$$

with $R_{0}>0$, with $R_{0}$ usually in [6 dB, $20 \mathrm{~dB}$ ]. This model is not $p s d$, as shown in the following example: let $N=7$ and all $r_{i}$ equal, and let $\vec{r}_{i}=0,15, \ldots, 90^{\circ}$.

The problem with the preceding model is its discontinuity at $\theta=60^{\circ}$. This is simple to resolve: if we use the general form (27) as $h_{\Theta}(\theta)$ and (31) as $h_{\mathrm{R}}(R)$, then we have a very flexible model that is always $p s d$.

Proof: $h_{\mathrm{R}}(R)$ is shown to be $p s d$ by choosing

$$
f(\omega)=\frac{2 \sin ^{2}\left(\pi R_{0} \omega\right)}{\pi^{2} R_{0} \omega^{2}}
$$

with $g(\vec{r})=(10 / \ln 10) \ln \|\vec{r}\|$ in Theorem 5. Equivalently, we may use Pólya's theorem, as in [56].

The model (27) was already shown to be $p s d$.

Now, $h$ is expressed as a product of two psd models, and it follows from Theorem 6 that $h(\theta, R)$ is $p s d$.

2) Angle-Absolute Distance: In [46], a separable model depending on $d$ and $\theta$ is used and can be rewritten as

$$
\begin{aligned}
h_{\mathrm{d}}(d) & =e^{-d / d_{0}} \\
h_{\Theta}(\theta) & = \begin{cases}1, & \theta \leq 90^{\circ} \\
0, & \theta>90^{\circ}\end{cases} \\
h(d, \theta) & =h_{\mathrm{d}}(d) h_{\Theta}(\theta) .
\end{aligned}
$$

(There is a typo in the original equation [46, eq. (18)]: the term $\sigma_{\bar{\psi}_{p}}^{2}$ should be removed, as confirmed in a private communication with D. Kaltakis).

The authors say that this model is $p s d$; however, this claim is not substantiated, although we understand that their simulations always gave $p s d$ correlation matrices. We already recognise the model $h_{\mathrm{d}}(d)$ from (19), which we know to be $p s d$. However, it is simple to show that $h_{\Theta}$ is non-psd; therefore, the product of the two might not be psd either. In fact, the following counterexample shows it is not $p s d$ : $N=14$ with $r_{i}=d_{0}$, and $\angle \vec{r}_{i}=0,180 / 7, \ldots, 2340 / 7^{\circ}$.

The authors of [46] claim that this model is psd based on their simulations. This is to be expected: because the model can be approximated by (19) for $r_{i} \gg d_{0}$, as might be the case in the cellular context, it can often appear $p s d$ in simulations. 
However, strictly speaking, it is non-psd, and our example shows that it can fail, particularly in cases of small $r_{i}$.

In [94], the same author proposes

$$
\begin{aligned}
h_{\mathrm{d}}(d) & =e^{-d / d_{0}} \\
h_{\Theta}(\theta) & =\max (0, \cos (\theta)) \\
h(d, \theta) & =h_{\mathrm{d}}(d) h_{\Theta}(\theta) .
\end{aligned}
$$

Again, while the model appears psd [94], in fact, it is not, as attested to by the following counterexample: $N=8$ with $r_{i}=0.4 d_{0}$ and $\angle \vec{r}_{i}=0,45, \ldots, 325^{\circ}$. However, this is a case with very small $r_{i}$. In practice, for $r_{i} \gg d_{0}$, this model is well approximated by

$$
h(d, \theta)=e^{-d / d_{0}} \cos (\theta)
$$

which is $p s d$.

Proof: Model (34) is the product of models (19) and (24) with $A=1$ and $B=0$, both of which were shown to be $p s d$. Then, by Theorem 6, (34) is psd.

\section{E. More Elaborate (Nonseparable) Models}

It has been suggested [56] that separable models might not be sufficient to accurately model shadowing correlation.

Saunders' model [73] has been used in [7], [43], [45], [57], and [95] (but watch for various transcription errors), i.e.,

$$
\begin{aligned}
h_{1} & =10^{-0.05 R} \\
h_{2} & = \begin{cases}1, & \theta<\theta_{\mathrm{T}} \\
\left(\theta_{T} / \theta\right)^{\gamma}, & \theta \geq \theta_{\mathrm{T}}\end{cases} \\
\theta_{\mathrm{T}} & =2 \arcsin \frac{d_{0}}{2 \min \left\{r_{1}, r_{2}\right\}} \\
h\left(\theta, r_{1}, r_{2}\right) & =h_{1} h_{2}
\end{aligned}
$$

where $d_{0}$ is the same decorrelation distance as measured for model (19), and $\gamma>0$ is the other tunable parameter (a typical value is $\gamma=0.3$ ). The model is not separable in $\theta$ and $R$ because $\theta_{\mathrm{T}}$ depends on $r_{1}$ and $r_{2}$. This model is non- $p s d$, as seen in [45], [46], and [57] and as is apparent from this example: $N=3$ with $r_{i}=r \geq d_{0} / \sqrt{2}$, and $\angle \vec{r}_{i}=0, \theta_{T}, 2 \theta_{T}$.

In addition, we see that this model is undefined for $r_{i}<$ $d_{0} / 2$, since the domain of $\arcsin$ is $[-1,1]$. To rectify this problem, Saunders' model has been modified [1] (also [88], but note some transcription errors) by augmenting its domain, i.e.,

$$
h\left(\theta, r_{1}, r_{2}\right)= \begin{cases}\text { same as }(35), & r_{1}, r_{2} \geq d_{0} / 2 \\ \sqrt{d_{0} / 2 \max \left\{r_{1}, r_{2}\right\}}, & \text { otherwise }\end{cases}
$$

Notice, however, that if more than one $r_{i}$ is less than $d_{0} / 2$, then we will again have $h>1$, which is not a valid correlation value.

In addition, extending the model's domain cannot make it $p s d$ : the same counterexamples used to show that (35) is non$p s d$ can be used to show that (36) is non-psd.
Another model was given in [33, Tab. II] and used in [45]. It is piecewise constant on rectangles in the $\theta-R$ domain, i.e.,

$$
h(\theta, R)=\left\{\begin{array}{cccc}
R<2, & 2<R<4, & R \geq 4 & \\
0.8, & 0.6, & 0.4, & \theta<30^{\circ} \\
0.5, & 0.4, & 0.2, & 30^{\circ}<\theta<60^{\circ} \\
0.4, & 0.4, & 0.2, & 60^{\circ}<\theta<90^{\circ} \\
0.2, & 0.2, & 0.2, & \theta \geq 90^{\circ} .
\end{array}\right.
$$

The entries of this table cannot be obtained from an outer product, and thus the model is nonseparable. The model is non- $p s d$, as observed in [45, 46, 57]; for example: $N=6$ with $r_{i}=r \forall i$ and $\angle \vec{r}_{i}=0,7,14,21,28,35^{\circ}$.

Another model is given in [22], i.e.,

$$
\begin{aligned}
h_{\Theta}(\theta) & \text { as in }(25) \\
h_{\mathrm{R}}(R) & =\max \left(0,\left(1-R / R_{0}\right)^{\alpha}\right) \\
h(\theta, R) & =h_{\mathrm{R}}(R)\left(h_{\Theta}(\theta)+a\right)+b
\end{aligned}
$$

with $a>0, b>0, a+b \leq 0.22, \alpha \geq 0$, and $R_{0}>0$. When $b \neq 0$, this model is not quite separable. It is not $p s d$, as seen from the same counterexample used for model (25), with $a=$ $b=0$ and $r_{i}=r \forall i$.

Finally, the model "1.0/0.4 RX" in [72] takes the following form:

$$
h(\theta, R)= \begin{cases}\max \left(0,1-\frac{R}{R_{0}}\right)\left(0.6-\frac{\theta}{150^{\circ}}\right)+0.4, & \theta \leq 60^{\circ} \\ 0.4, & \theta>60^{\circ}\end{cases}
$$

where $R_{0}$ is chosen between 6 and $20 \mathrm{~dB}$. This model is not $p s d$, as attested to by the following example: with $N=4$, regardless of the value of $R_{0}$, choose $r_{i}$ all equal, and $\angle \vec{r}_{i}=0,30,60,90^{\circ}$.

\section{Some Thoughts on Physical Realism}

While this paper focuses on the mathematical feasibility of correlation models, we would like to give a few thoughts about physical realism as well: can the correlation model $h$ be considered realistic based on what we know about wireless propagation? Does it make sense intuitively? It is important to keep in mind that, while mathematical feasibility is an objective criterion, physical plausibility is relative to the understanding of wireless shadowing, and therefore, the criteria presented in this section are merely tentative. We hope that these initial ideas will encourage thought and discussion on what a shadowing correlation model should realistically look like. Nevertheless, these initial criteria can give a first approximation in choosing a good correlation model.

Proposition 1: Shadowing is a large-scale phenomenon, averaged over small displacements in space (and time), and should not by nature vary quickly in these dimensions [26], [45], [56], [62].

Proposition 2: As argued in [7], [18], [37], [59], [60], [72], [73], [86], [95], and [96], correlation in shadowing may be explained by a partial overlap of the large-scale propagation medium, as seen in Fig. 4. The nonoverlapping propagation areas are considered independent. When a propagation front 


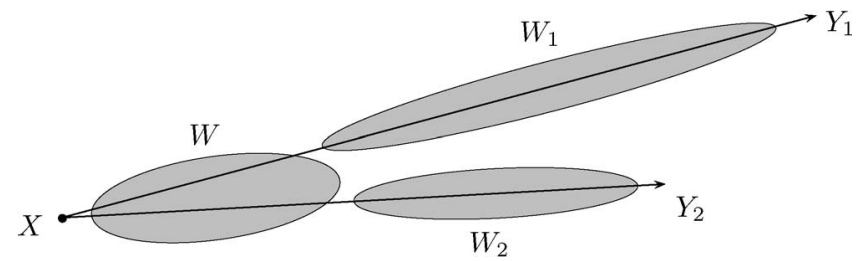

Fig. 4. Physical argument of common propagation area for correlation in shadowing.

passes through successive areas, the gains are multiplicative and thus add in the logarithmic domain. We may then write the following:

Let $W, W_{1}$, and $W_{2}$ be independent random variables with zero mean and variances $a^{2}, b^{2}$, and $c^{2}$, respectively. We have

$$
\begin{aligned}
& S_{1}=W+W_{1} \\
& S_{2}=W+W_{2} .
\end{aligned}
$$

It follows that

$$
\begin{aligned}
\mathbb{V A R}\left\{S_{1}\right\} & =a^{2}+b^{2}=\sigma_{\mathrm{s}}^{2}\left(\vec{r}_{1}\right) \\
\mathbb{V A R}\left\{S_{2}\right\} & =a^{2}+c^{2}=\sigma_{\mathrm{s}}^{2}\left(\vec{r}_{2}\right) \\
\mathbb{E}\left\{S_{1} S_{2}\right\} & =a^{2}
\end{aligned}
$$

and therefore

$$
h\left(\vec{r}_{1}, \vec{r}_{2}\right)=\frac{a^{2}}{\sqrt{\left(a^{2}+b^{2}\right)\left(a^{2}+c^{2}\right)}} .
$$

This applies to any pair of shadowing terms.

This interpretation leads us to formulate the following criteria.

1) $h\left(\vec{r}_{i}, \vec{r}_{j}\right) \approx 1$ for $\vec{r}_{i} \approx \vec{r}_{j}$.

2) $h\left(\vec{r}_{i}, \vec{r}_{j}\right) \ll 1$ for $\left\|\vec{r}_{i}-\vec{r}_{j}\right\| \gg 0$.

3) $h$ should be a nonincreasing function in $\theta$ [24], [33], [45], [56] $R$ [33] and $d$.

4) $h$ should be nonnegative, according to (41). However, this is contradicted by some measurement campaigns [24], [26], [27], [30], [34], [36]-[39], which reported some significantly negative estimated correlation coefficients in some cases. It would be interesting to study whether these observations are statistically significant. In addition, we suspect that negative correlations in measurements may appear in a particular obstacle scenario. However, averaged over very many obstacle realisations, the correlations are perhaps less likely to be negative.

5) $h$ should be small for large $\theta$ and approach zero for $\theta \approx$ $180^{\circ}$, and $r_{1}$ and $r_{2}$ large, as the propagation regions are then essentially nonoverlapping.

6) Continuity: a small change in $\vec{r}_{i}$ should result in small changes in $h\left(\vec{r}_{i}, \vec{r}_{j}\right)$, at least when $r_{i}$ is large.

7) Correlation should not depend on $d$ only, as the distance between $\vec{r}_{1}$ and $\vec{r}_{2}$ tells us little about the overlap between the corresponding propagation areas.

Furthermore, it was argued [37], [38], [45], [56], [62], [72] that cross-correlation should depend on $\theta$ and $R$.
We have checked each model against these physical constraints and summarised the results (without proof) in Table I. The only model that is both $p s d$ and fulfills all these physical criteria is the model we proposed based on [72], as given by (27), (29), and (31), with $a=1$, and $b=0$.

\section{Feasibility ACCORDing to MARGinAl Distribution}

Until now, we have only verified whether a given model $h$ gives $p s d$ covariance matrices $\mathbf{K}$. This is a necessary but not sufficient condition to ensure that a random vector $\mathbf{S}$ can be constructed with covariance $\mathbf{K}$. To further study feasibility, it is necessary to look at the marginal distribution of $S_{i}$.

\section{A. Jointly Lognormal Shadowing}

In the most common case of lognormal shadowing (i.e., each $S_{i}$ being Gaussian), a natural and effective way to jointly model shadowing paths is by making $S_{1}, \ldots, S_{N}$ jointly Gaussian. We then say that shadowing is jointly lognormal. In this case, every symmetric $p s d$ correlation matrix is feasible by the following explicit construction.

We begin by solving for $\mathbf{C}_{N \times N}$ in

$$
\mathbf{K}=\mathbf{C C}^{\mathrm{T}} \text {. }
$$

In general, there are many solutions to this equation if and only if $\mathbf{K}$ is $p s d$. Then, we generate an independent column vector of standard Gaussian random variables $\mathbf{Z}=\left[Z_{1}, \ldots, Z_{N}\right]$ and obtain the shadowing terms with

$$
\mathbf{S}=\mathbf{C Z}
$$

There exist various algorithms for calculating C. In the case when $\mathbf{K}$ is positive definite, Cholesky factorization is a stable algorithm [57], [97] that gives a triangular solution for C. However, $\mathbf{K}$ is allowed to be psd in general. ${ }^{3}$ In addition, numerical rounding can make $\mathbf{K}$ slightly non- $p s d$. To fix this double problem, there are at least two procedures. One is to slightly modify $\mathbf{K}$ so that it becomes positive definite [57]. However, $\mathbf{K}$ need not be positive definite (as suggested in [43], [56], and [57]) but may be psd in general. In this case, Cholesky factorization is not applicable, and matrix diagonalization (eigenvalue decomposition) [1], [7], [58], [95] should be used. In addition, should some of the resulting eigenvalues of $\mathbf{K}$ be slightly negative due to rounding, they can be set to zero [58]. Then, if there are eigenvalues equal to zero, $\mathbf{K}$ is not full rank and therefore not positive definite, but $\mathbf{C}$ can still be found. Additionally, if $\mathbf{K}$ is highly correlated, then a fast approximation exists [98].

We may conclude that, for lognormal shadowing, it is only necessary for a model $h$ to be $p s d$ for it to be possible to explicitly construct any number of correlated shadowing paths.

\footnotetext{
${ }^{3}$ The case when $\mathbf{K}$ is $p s d$ but not positive definite corresponds to a singular matrix. In practice, for randomly generated positions $\vec{r}_{i}$ and using doubleprecision arithmetic, we find this event to be extremely rare [13], [14], and thus, Cholesky factorization is almost safe.
} 


\section{B. Non-Lognormal Shadowing}

While lognormal is a well-established [8], [27], [73], [78], [86] and by far the most common model for the marginal distribution of shadowing, it is not the only model available. Other models have been proposed, either from physical plausibility arguments or for easier mathematical tractability. The two other models that we have encountered for the distribution of $e^{S_{i}}$ are truncated lognormal [86], [99] and Gamma [100]-[102]. See [86, Sec. V-B4] for a survey of shadowing distribution measurements.

We have seen in Section III that the condition for the finite variance of $S_{i}$ is required for an adequate definition of correlation. For the truncated lognormal model, which corresponds to a truncated Gaussian $S_{i}$, it is easy to see that truncation reduces the variance; hence, it is finite. For Gamma shadowing, it is easy to verify that all the moments of the logarithm of a Gamma random variable are finite, since they can be expressed as the integral in [103, Integral 4.352.1].

It is, however, not evident that the vector $\mathbf{S}$ can be generated according to non-Gaussian marginal distributions and a given psd correlation matrix $\mathbf{K}$. One constructive method for obtaining such jointly distributed vectors is Normal-to-Anything (NORTA), as described in [104]. This method requires some more stringent conditions on the marginal distributions to be feasible. These conditions might merit further study should non-lognormal shadowing models gain popularity.

\section{CONClusion}

We propose the criteria developed in this paper as a basis for evaluating, designing, and correcting existing future correlation models. Naturally, there is more to be said both on testing more complex models for being $p s d$ and on their physical plausibility.

\section{A. For Those Wanting to Incorporate Shadowing Correlation}

For lognormal shadowing, only certain models that have the psd property (see Table I) guarantee to always give feasible joint shadowing distributions. For non-lognormal shadowing, some additional conditions might be required.

Based on both mathematical feasibility and physical arguments, we conclude that a subset of the family of models inspired by [72] is the best existing candidate for modeling correlation in shadowing. This model is given by (29), (31), and (27) with $a=1$ and $b=0$. With two tunable parameters $\theta_{0}$ and $R_{0}$, it may be tuned to approximate many other correlation models that might have less-desirable properties.

\section{B. For Those Using a Model That Is Not Feasible}

Models that are not psd may still be used in particular application scenarios. For example, all models give psd correlation matrices for $N=2$. In addition, we have argued that a correlation model that exactly corresponds to reality must per force be $p s d$. It follows that for any model that approximates reality well (whether from extensive measurement campaigns or from theoretical arguments), there exists a model that is close to it (according to some reasonable metric) while also being $p s d$. For example, the authors in [72] fit the $p s d$ model (27) to the data of [24] to replace the non-psd model (25).

Our suggestion is to take non-psd models and slightly correct them to make them psd, as we have done for (33).

\section{For Those Designing New Correlation Models}

We suggest that all new correlation models be designed as psd for the reasons described in Section I-A. Theorem 6 can be used to construct more detailed separable models.

\section{ACKNOWLEDGMENT}

The authors would like to thank the following people for valuable discussions that helped form this paper: Dr. F. Graziosi and Dr. L. Imbriglio (University of L'Aquila, L'Aquila, Italy), Dr. M. Di Renzo (University of Edinburgh, Edinburgh, U.K.), Dr. N. Cardona and Dr. J. Monserrat (Polytechnic University of Valencia, Valencia, Spain), and R.-A. Pitaval (Aalto University, Espoo and Helsinki, Finland).

\section{REFERENCES}

[1] D. Kitchener, M. Naden, W. Tong, P. Zhu, G. Senarnath, H. Zhang, D. Steer, and D. Yu, "Correlated lognormal shadowing model," San Diego, CA, IEEE 802.16 Session 44, Tech. Rep., Jul. 2006.

[2] N. Patwari and P. Agrawal, "Effects of correlated shadowing: connectivity, localization, and RF tomography," in Proc. IEEE IPSN, Apr. 2008, pp. 82-93.

[3] B. Kasiri, M. Naderi, and B. Abolhassani, "A new realistic relay selection method based on correlated shadowing for multihop cellular networks," in Proc. IEEE ICCEE, Dec. 2008, pp. 669-673.

[4] F. Fabbri and R. Verdone, "The impact of correlated channel fluctuations on the connectivity of wireless ad-hoc networks," in Proc. IEEE VTC, Apr. 2009, pp. 1-5.

[5] J. H. Lee and R. M. Buehrer, "Location estimation using differential RSS with spatially correlated shadowing," in Proc. IEEE GLOBECOM, Nov. 2009, pp. 1-6.

[6] F. Graziosi, M. Pratesi, M. Ruggieri, and F. Santucci, "A multicell model of handover initiation in mobile cellular networks," IEEE Trans. Veh. Technol., vol. 48, no. 3, pp. 802-814, May 1999.

[7] X. Yang, S. Ghaheri-Niri, and R. Tafazolli, "Downlink soft handover gain in CDMA cellular network with cross-correlated shadowing," in Proc. IEEE VTC, Oct. 2001, vol. 1, pp. 276-280.

[8] F. Graziosi and F. Santucci, "A general correlation model for shadow fading in mobile radio systems," IEEE Commun. Lett., vol. 6, no. 3, pp. 102-104, Mar. 2002.

[9] J. Kim and G. Stüber, "CDMA soft handoff analysis in the presence of power control error and shadowing correlation," IEEE Trans. Wireless Commun., vol. 1, no. 2, pp. 245-255, Apr. 2002.

[10] L.-C. Wang, G. Stuber, and C.-T. Lea, "Effects of Rician fading and branch correlation on a local-mean-based macrodiversity cellular system," IEEE Trans. Veh. Technol., vol. 48, no. 2, pp. 429-436, Mar. 1999.

[11] J. Zhang and V. Aalo, "Effect of macrodiversity on average-error probabilities in a Rician fading channel with correlated lognormal shadowing," IEEE Trans. Commun., vol. 49, no. 1, pp. 14-18, Jan. 2001.

[12] M. DeFaria and E. Sousa, "Effect of intercell interference on the SNIR of a multihop cellular network," in Proc. IEEE VTC, May/Jun. 2005, vol. 5, pp. 3107-3111.

[13] S. S. Szyszkowicz and H. Yanikomeroglu, "Analysis of interference from large clusters as modeled by the sum of many correlated lognormals," in Proc. IEEE WCNC, Mar./Apr. 2008, pp. 741-745.

[14] S. S. Szyszkowicz, F. Alaca, H. Yanikomeroglu, and J. S. Thompson, "Efficient simulation using shadowing fields of many wireless interferers with correlated shadowing," in Proc. IEEE VTC, May 2010, pp. 1-5.

[15] N. Gogate, D. Avidor, and S. Panwar, "Simulation study of a fixed wireless access system," in Proc. IEEE ICUPC, Oct. 1998, vol. 1, pp. 209-215. 
[16] H. Haas, S. McLaughlin, and G. Povey, "An investigation on capacity versus guard-bands in the TDD mode of UMTS," in Proc. IEEE VTC, Sep. 2000, vol. 4, pp. 1820-1824.

[17] D. Avidor, N. Hegde, and S. Mukherjee, "On the impact of the soft handoff threshold and the maximum size of the active group on resource allocation and outage probability in the UMTS system," IEEE Trans. Wireless Commun., vol. 3, no. 2, pp. 565-577, Mar. 2004.

[18] H. Arnold, D. Cox, and R. Murray, "Macroscopic diversity performance measured in the $800-\mathrm{MHz}$ portable radio communications environment," IEEE Trans. Antennas Propag., vol. 36, no. 2, pp. 277-281, Feb. 1988.

[19] S. Mukherjee and D. Avidor, "Effect of macrodiversity and microdiversity on outages and bit error rates in a cellular system," in Proc. IEEE WCNC, Sep. 2000, vol. 2, pp. 649-654.

[20] W. Hwang, H. Kang, Y. Choi, and K. Kim, "Effects of correlated shadowing and multipath fading on OFDM systems with postdetectioncombining-based macrodiversity," in Proc. WPMC, Oct. 2002, vol. 2, pp. 449-453.

[21] S. Mukherjee and D. Avidor, "Effect of microdiversity and correlated macrodiversity on outages in a cellular system," IEEE Trans. Wireless Commun., vol. 2, no. 1, pp. 50-58, Jan. 2003.

[22] Y. Chen, L. Hu, C. Yuen, Y. Zhang, Z. Zhang, and P. Rapajic, "Intrinsic measure of diversity gains in generalised distributed antenna systems with cooperative users," IET Commun., vol. 3, no. 2, pp. 209-222, Feb. 2009.

[23] C.-X. Wang, X. Hong, X. Ge, X. Cheng, G. Zhang, and J. Thompson, "Cooperative MIMO channel models: a survey," IEEE Commun. Mag., vol. 48, no. 2, pp. 80-87, Feb. 2010.

[24] V. Graziano, "Propagation correlations at $900 \mathrm{MHz}$," IEEE Trans. Veh. Technol., vol. VT-27, no. 4, pp. 182-189, Nov. 1978.

[25] M. J. Marsan, G. C. Hess, and S. S. Gilbert, "Shadowing variability in an urban land mobile environment at $900 \mathrm{MHz}$," Electron. Lett., vol. 26, no. 10, pp. 646-648, May 1990.

[26] M. Gudmundson, "Correlation model for shadow fading in mobile radio systems," Electron. Lett., vol. 27, no. 23, pp. 2145-2146, Nov. 1991.

[27] J. Liberti and T. Rappaport, "Statistics of shadowing in indoor radio channels at 900 and $1900 \mathrm{MHz}$," in Proc. IEEE MILCOM, Oct. 1992, vol. 3, pp. 1066-1070.

[28] A. Mawira, "Models for the spatial correlation functions of the (log)normal component of the variability of VHF/UHF field strength in urban environment," in Proc. IEEE PIMRC, Oct. 1992, pp. 436-440.

[29] R. Stevens and I. Dilworth, "Mobile radio shadowing loss variability and co-channel signal correlation at $452 \mathrm{MHz}$," Electron. Lett., vol. 32, no. 1, pp. 16-17, Jan. 1996.

[30] K. Butterworth, K. Sowerby, and A. Williamson, "Correlated shadowing in an in-building propagation environment," Electron. Lett., vol. 33, no. 5, pp. 420-422, Feb. 1997.

[31] P. Taaghol and R. Tafazolli, "Correlation model for shadow fading in land-mobile satellite systems," Electron. Lett., vol. 33, no. 15, pp. 12871289, Jul. 1997.

[32] T. Sorensen, "Correlation model for slow fading in a small urban macro cell," in Proc. IEEE PIMRC, Sep. 1998, vol. 3, pp. 1161-1165.

[33] K. Zayana and B. Guisnet, "Measurements and modelisation of shadowing cross-correlations between two base-stations," in Proc. IEEE ICUPC, Oct. 1998, vol. 1, pp. 101-105.

[34] T. Sorensen, "Slow fading cross-correlation against azimuth separation of base stations," Electron. Lett., vol. 35, no. 2, pp. 127-129, Jan. 1999.

[35] E. Perahia and D. Cox, "Shadow fading correlation between uplink and downlink," in Proc. IEEE VTC, May 2001, vol. 1, pp. 308-312.

[36] E. Perahia, D. Cox, and S. Ho, "Shadow fading cross correlation between basestations," in Proc. IEEE VTC, May 2001, vol. 1, pp. 313-317.

[37] J. Weitzen and T. Lowe, "Measurement of angular and distance correlation properties of log-normal shadowing at $1900 \mathrm{MHz}$ and its application to design of PCS systems," IEEE Trans. Veh. Technol., vol. 51, no. 2, pp. 265-273, Mar. 2002.

[38] N. Jalden, P. Zetterberg, L. Garcia, and B. Ottersten, "Inter- and intrasite correlation of large-scale parameters from macrocellular measurements at $1800 \mathrm{MHz}$," EURASIP J. Wireless Commun. Netw., vol. 2007, no. 3, p. 10, Jul. 2007.

[39] N. Jalden, P. Zetterberg, and B. Ottersten, "Directional dependence of large scale parameters in wireless channel models," in Proc. IEEE WCNC, Mar./Apr. 2008, pp. 1223-1228.

[40] P. Dassanayake, "Spatial correlation of shadow fading and its impact on handover algorithm parameter settings," in Proc. IEEE Netw. Conf., Jul. 1995, pp. 225-229.

[41] G. Malmgren, "On the performance of single frequency networks in correlated shadow fading," IEEE Trans. Broadcast., vol. 43, no. 2, pp. 155-165, Jun. 1997.
[42] R. Fraile, J. F. Monserrat, J. Gozalvez, and N. Cardona, "Mobile radio bi-dimensional large-scale fading modelling with site-to-site crosscorrelation," Eur. Trans. Telecommun., vol. 19, no. 1, pp. 101-106, Jan./Feb. 2008.

[43] J. F. Monserrat, R. Fraile, D. Calabuig, and N. Cardona, "Complete shadowing modeling and its effect on system level performance evaluation," in Proc. IEEE VTC, May 2008, pp. 294-298.

[44] B. Kasiri, M. Naderi, and B. Abolhassani, "The effect of correlated link shadowing on the performance of multihop cellular networks," in Proc. IEEE ICCEE, Dec. 2008, pp. 951-955.

[45] R.-A. Pitaval, "Relay deployment in single frequency network," M.S. thesis, Helsinki Univ. Technol., Espoo, Finland, Aug. 2009.

[46] D. Kaltakis, M. A. Imran, and R. Hoshyar, "Uplink capacity with correlated lognormal shadow fading," in Proc. IEEE VTC, Apr. 2009, pp. 1-5.

[47] M. Di Renzo, F. Graziosi, and F. Santucci, "Cooperative spectrum sensing in cognitive radio networks over correlated log-normal shadowing," in Proc. IEEE VTC, Apr. 2009, pp. 1-5.

[48] W. Lee and D.-H. Cho, "A new neighbor discovery scheme based on spatial correlation of wireless channel," in Proc. IEEE VTC, Apr. 2009, pp. $1-5$.

[49] A. Sibille, "Efficient generation of spatially and frequency correlated random values for cognitive radio network simulators," IEEE Trans. Veh. Technol., vol. 59, no. 3, pp. 1121-1128, Mar. 2010.

[50] D. Kotz, C. Newport, and C. Elliott, "The mistaken axioms of wirelessnetwork research," Dept. Comput. Sci., Dartmouth College, Hanover, NH, Tech. Rep. Dartmouth TR2003-467, Jul. 2003.

[51] K.-J. Yang and Y.-R. Tsai, "Location tracking in mobile networks under correlated shadowing effects," in Proc. IEEE WCNC, Apr. 2009, pp. $1-5$.

[52] A. Ghasemi and E. Sousa, "Asymptotic performance of collaborative spectrum sensing under correlated log-normal shadowing," IEEE Commun. Lett., vol. 11, no. 1, pp. 34-36, Jan. 2007.

[53] A. Ghasemi and E. S. Sousa, "Fundamental limits of spectrum-sharing in fading environments," IEEE Trans. Wireless Commun., vol. 6, no. 2, pp. 649-658, Feb. 2007.

[54] M. Di Renzo, L. Imbriglio, F. Graziosi, and F. Santucci, "Cooperative spectrum sensing over correlated log-normal sensing and reporting channels," in Proc. IEEE GLOBECOM, Nov. 2009, pp. 1-8.

[55] F. Graziosi, M. Pratesi, M. Ruggieri, and F. Santucci, "Modeling of handover initiation algorithms with correlated co-channel interferers," in Proc. IEEE ICUPC, Oct. 1997, pp. 244-248.

[56] D. Avidor and S. Mukherjee, "Hidden issues in the simulation of fixed wireless systems," Wireless Netw., vol. 7, no. 2, pp. 187-200, Mar. 2001

[57] J. F. Monserrat, R. Fraile, and L. Rubio, "Application of alternating projection method to ensure feasibility of shadowing cross-correlation models," Electron. Lett., vol. 43, no. 13, pp. 724-725, Jun. 2007.

[58] P. J. Rousseeuw and G. Molenberghs, "Transformation of non positive semidefinite correlation matrices," Commun. Stat. A-Theory Methods, vol. 22, no. 4, pp. 965-984, 1993.

[59] A. J. Viterbi, A. M. Viterbi, and E. Zehavi, "Other-cell interference in cellular power-controlled CDMA," IEEE Trans. Commun., vol. 42, no. 2-4, pp. 1501-1504, Feb.-Apr. 1994.

[60] A. Viterbi, A. Viterbi, K. Gilhousen, and E. Zehavi, "Soft handoff extends CDMA cell coverage and increases reverse link capacity," IEEE J. Sel. Areas Commun., vol. 12, no. 8, pp. 1281-1288, Oct. 1994.

[61] F. Graziosi, "Analysis of outage duration for mobile radio networks with correlated shadowings," in Proc. IEEE VTC, May 1999, vol. 2, pp. 948-952.

[62] S. Mukherjee and D. Avidor, "Dynamics of path losses between a mobile terminal and multiple base stations in a cellular environment," IEEE Trans. Veh. Technol., vol. 50, no. 6, pp. 1590-1603, Nov. 2001.

[63] D. Avidor and S. Mukherjee, "Downlink dimensioning for the HSDPA standard," Wireless Pers. Commun., vol. 32, no. 2, pp. 139-152, Jan. 2005.

[64] C. Fischione, F. Graziosi, and F. Santucci, "Approximation for a sum of on-off lognormal processes with wireless applications," IEEE Trans. Commun., vol. 55, no. 10, pp. 1984-1993, Oct. 2007.

[65] D. S. Bernstein, Matrix Mathematics: Theory, Facts, and Formulas With Application to Linear Systems Theory. Princeton, NJ: Princeton Univ. Press, 2005.

[66] A. M. Yaglom, Correlation Theory of Stationary and Related Random Functions: Volume 1: Basic Results. Berlin, Germany: SpringerVerlag, 1987.

[67] Z. Wang, E. Tameh, and A. Nix, "Joint shadowing process in urban peer-to-peer radio channels," IEEE Trans. Veh. Technol., vol. 57, no. 1, pp. 52-64, Jan. 2008. 
[68] P. Agrawal and N. Patwari, "Correlated link shadow fading in multihop wireless networks," IEEE Trans. Wireless Commun., vol. 8, no. 8, pp. 4024-4036, Aug. 2009.

[69] C. Oestges and D. Vanhoenacker-Janvier, "A physical-statistical shadowing correlation model and its application to low-Earth-orbit systems," IEEE Trans. Veh. Technol., vol. 50, no. 2, pp. 416-421, Mar. 2001.

[70] O. Ekici and A. Yongacoglu, "The effect of correlated shadowing on power control error in satellite CDMA systems," in Proc. IEEE VTC, May/Jun. 2005, vol. 4, pp. 2643-2647.

[71] C. Oestges and D. Vanhoenacker-Janvier, "Time series-based prediction of fade dynamics and shadowing correlation statistics for navigation systems," in Proc. EuCAP, Nov. 2007, pp. 1-6.

[72] T. Klingenbrunn and P. Mogensen, "Modelling cross-correlated shadowing in network simulations," in Proc. IEEE VTC, Sep. 1999, vol. 3, pp. 1407-1411.

[73] S. Saunders and A. Aragón-Zavala, Antennas and Propagation for Wireless Communication Systems, 2nd ed. New York: Wiley, 2007.

[74] K. Yamamoto, A. Kusuda, and S. Yoshida, "Impact of shadowing correlation on coverage of multihop cellular systems," in Proc. IEEE ICC, Jun. 2006, vol. 10, pp. 4538-4542.

[75] R. Fraile, J. Nasreddine, N. Cardona, and X. Lagrange, "Multiple diffraction shadowing simulation model," IEEE Commun. Lett., vol. 11, no. 4, pp. 319-321, Apr. 2007.

[76] H. Kim and Y. Han, "Enhanced correlated shadowing generation in channel simulation," IEEE Commun. Lett., vol. 6, no. 7, pp. 279-281, Jul. 2002.

[77] Z. Lin, X. Peng, K. B. Png, and F. Chin, "Kronecker modelling for correlated shadowing in UWB MIMO channels," in Proc. IEEE WCNC, Mar. 2007, pp. 1583-1587.

[78] A. Goldsmith, L. Greenstein, and G. Foschini, "Error statistics of real-time power measurements in cellular channels with multipath and shadowing," IEEE Trans. Veh. Technol., vol. 43, no. 3, pp. 439-446, Aug. 1994.

[79] N. B. Mandayam, P.-C. Chen, and J. M. Holtzman, "Minimum duration outage for cellular systems: a level crossing analysis," in Proc. IEEE VTC, Apr. 1996, vol. 2, pp. 879-883.

[80] R. Vijayan and J. Holtzman, "A model for analyzing handoff algorithms," IEEE Trans. Veh. Technol., vol. 42, no. 3, pp. 351-356, Aug. 1993.

[81] D. Giancristofaro, "Correlation model for shadow fading in mobile radio channels," Electron. Lett., vol. 32, no. 11, pp. 958-959, May 1996.

[82] A. A. Abu-Dayya and N. C. Beaulieu, "Outage probabilities in the presence of correlated lognormal interferers," IEEE Trans. Veh. Technol., vol. 43, no. 1, pp. 164-173, Feb. 1994.

[83] S. S. Szyszkowicz and H. Yanikomeroglu, "Limit theorem on the sum of identically distributed equally and positively correlated joint lognormals," IEEE Trans. Commun., vol. 57, no. 12, pp. 3538-3542, Dec. 2009.

[84] M. Pratesi, M. Ruggieri, F. Graziosi, and F. Santucci, "Performance of signal strength handover algorithms with interference and correlated shadowings," in Proc. IEEE VTC, May 1997, vol. 2, pp. 530-534.

[85] D. Baum, J. Hansen, and J. Salo, "An interim channel model for beyond$3 \mathrm{G}$ systems: extending the $3 \mathrm{GPP}$ spatial channel model (SCM)," in Proc. IEEE VTC, May/Jun. 2005, vol. 5, pp. 3132-3136.

[86] J. Salo, L. Vuokko, H. M. El-Sallabi, and P. Vainikainen, "An additive model as a physical basis for shadow fading," IEEE Trans. Veh. Technol., vol. 56, no. 1, pp. 13-26, Jan. 2007.

[87] I.-K. Fu, C.-F. Li, T.-C. Song, and W.-H. Sheen, Correlation Models for Shadow Fading Simulation, IEEE Std. C802.16m-07/060, Tech. Rep., Mar. 2007.

[88] W. Ni, W. Zou, and H. Wang, "Modeling of spatially cross-correlated shadow fading in distributed radio access networks," in Proc. IEEE ICC, May 2008, pp. 4472-4476.

[89] N. B. Mehta, A. F. Molisch, J. Wu, and J. Zhang, "Approximating the sum of correlated lognormal or, lognormal-Rice random variables," in Proc. IEEE ICC, Jun. 2006, vol. 4, pp. 1605-1610.

[90] A. Algans, K. Pedersen, and P. Mogensen, "Experimental analysis of the joint statistical properties of azimuth spread, delay spread, and shadow fading," IEEE J. Sel. Areas Commun., vol. 20, no. 3, pp. 523-531, Apr. 2002.

[91] D. Catrein and R. Mathar, "Gaussian random fields as a model for spatially correlated log-normal fading," in Proc. IEEE ATNAC, Dec. 2008, pp. 153-157.

[92] M. Haleem, D. Avidor, and R. Valenzuela, "Fixed wireless access system with autonomous resource assignment," in Proc. IEEE PIMRC, Sep. 1998, vol. 3, pp. 1438-1442.
[93] K. Sayrafian-Pour and D. Kaspar, "A novel model-based indoor positioning using signal strength," in Proc. IEEE PIMRC, Sep. 2007, pp. 1-5.

[94] D. Kaltakis, "Fundamental limits of GCMAC with fading," Ph.D. dissertation, Univ. Surrey, Surrey, U.K., Aug. 2009.

[95] X. Yang and R. Tafazolli, "A method of generating cross-correlated shadowing for dynamic system-level simulators," in Proc. IEEE PIMRC, Sep. 2003, vol. 1, pp. 638-642.

[96] S. Saunders and B. Evans, "The spatial correlation of shadow fading in macrocellular mobile radio systems," in Proc. IEE Colloq. Propag. Aspects Future Mobile Syst., Oct. 1996, pp. 2/1-2/6.

[97] N. J. Higham, "Analysis of the Cholesky decomposition of a semidefinite matrix," in Reliable Numerical Computation. Oxford, U.K.: Oxford Univ. Press, 1990, pp. 161-185.

[98] J. Geist, "Computer generation of correlated Gaussian random variables," Proc. IEEE, vol. 67, no. 5, pp. 862-863, May 1979.

[99] B. Hochwald, T. Marzetta, and V. Tarokh, "Multiple-antenna channel hardening and its implications for rate feedback and scheduling," IEEE Trans. Inf. Theory, vol. 50, no. 9, pp. 1893-1909, Sep. 2004.

[100] I. Kostic, "Analytical approach to performance analysis for channel subject to shadowing and fading," Proc. Inst. Elect. Eng.-Commun., vol. 152 , no. 6 , pp. 821-827, Dec. 2005.

[101] A. Abdi and M. Kaveh, "On the utility of gamma PDF in modeling shadow fading (slow fading)," in Proc. IEEE VTC, Jul. 1999, vol. 3, pp. 2308-2312.

[102] P. Shankar, "Macrodiversity and microdiversity in correlated shadowed fading channels," IEEE Trans. Veh. Technol., vol. 58, no. 2, pp. 727-732, Feb. 2009.

[103] I. S. Gradshteyn and I. M. Ryzhik, Tables of Integrals, Series, and Products, 7th ed. New York: Academic, 2007.

[104] M. C. Cario and B. L. Nelson, "Modeling and generating random vectors with arbitrary marginal distributions and correlation matrix," Dept. Ind. Eng. Manage. Sci., Northwestern Univ., Evanston, IL, Tech. Rep., Apr. 1997.

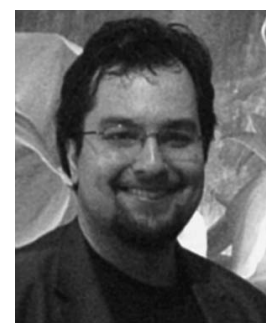

Sebastian S. Szyszkowicz (S'09) received the B.A.Sc. degree in electrical engineering (specializing in communications) in 2003 from the University of Ottawa, Ottawa, ON, Canada, and the M.A.Sc. degree wireless communications in 2007 from Carleton University, Ottawa, where he is currently working toward the Ph.D. degree in wireless communications.

In the winter of 2008, he was a Visiting Researcher with the University of Edinburgh, Edinburgh, U.K. He has given several invited talks about his research at universities in Europe and Canada. His research focuses on wireless interference and lognormal shadowing and the mathematical challenges therein.

Mr. Szyszkowicz was the recipient of the National Sciences and Engineering Research Council of Canada Postgraduate Scholarship at the Masters and Ph.D. levels. 


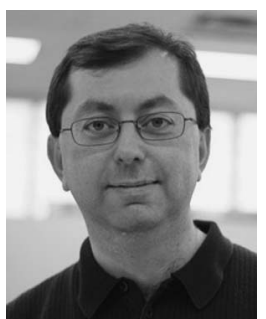

Halim Yanikomeroglu (M'98) received the B.Sc. degree in electrical and electronics engineering from the Middle East Technical University, Ankara, Turkey, in 1990 and the M.A.Sc. degree in electrical engineering (now ECE) and the Ph.D. degree in electrical and computer engineering from the University of Toronto, Toronto, ON, Canada, in 1992 and 1998, respectively.

He was with the Research and Development Group, Marconi Kominikasyon A.S., Ankara, from January 1993 to July 1994. Since 1998, he has been with the Department of Systems and Computer Engineering, Carleton University, Ottawa, ON, where he is currently a Full Professor with tenure. At Carleton University, he teaches graduate courses on digital, mobile, and wireless communications. In recent years, his research has been funded by Research In Motion (Canada), Huawei (China), Samsung (Korea), Nortel (Canada), Communications Research Centre Canada, and the National Sciences and Engineering Research Council (NSERC) of Canada. He has coauthored around 100 papers over the last five years. His research interests cover many aspects of the physical, medium access, and networking layers of wireless communications with a special emphasis on multihop/relay/mesh networks and cooperative communications.

Dr. Yanikomeroglu is a member of the Carleton University Senate. He is also a registered Professional Engineer in the province of Ontario. He was the recipient of the Carleton University Research Achievement Award in 2009 and the Carleton University Graduate Students Association Excellence Award in Graduate Teaching in 2010. He is Carleton University's nominee for the NSERC Steacie Fellowship for 2011. He has been involved in the steering committees and technical program committees of numerous international conferences in communications. He has also given about 20 tutorials at such conferences. He is a member of the Steering Committee of the IEEE Wireless Communications and Networking Conference (WCNC) and has been involved in the organization of this conference over the years, including serving as the Technical Program Cochair of WCNC 2004 and the Technical Program Chair of WCNC 2008. He was the General Cochair of the IEEE Vehicular Technology Conference held in Ottawa in September 2010. He was an Editor for the IEEE TRANSACTIONS ON WiRELESS COMMUNICATIONS (2002-2005) and IEEE COMMUNICATIONS SuRVEYS AND TUTORIALS (2002-2003), as well as a Guest Editor for the Wiley Journal on Wireless Communications and Mobile Computing. He was an Officer of the IEEE Technical Committee on Personal Communications (Chair: 2005-2006, Vice-Chair: 2003-2004, Secretary: 2001-2002), and he was also a member of the IEEE Communications Society's Technical Activities Council (2005-2006). He is an Adjunct Professor with King Saud University's Advanced Technology Research Center (Riyadh, Saudi Arabia).

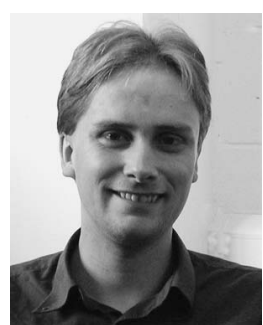

John S. Thompson (M'03) received the B.Eng. and $\mathrm{Ph} . \mathrm{D}$. degrees from the University of Edinburgh, Edinburgh, U.K., in 1992 and 1996, respectively.

From July 1995 to August 1999, he was a Postdoctoral Researcher with the University of Edinburgh, funded by the U.K. Engineering and Physical Sciences Research Council and Nortel Networks. Since September 1999, he has been a Lecturer with the School of Engineering and Electronics, University of Edinburgh. In October 2005, he was promoted to the position of Reader. He has published approximately 160 papers to date, including a number of invited papers, book chapters, and tutorial talks, as well as coauthoring an undergraduate textbook on digital signal processing. His research interests currently include signal processing algorithms for wireless systems, antenna array techniques, and multihop wireless communications.

Dr. Thompson is the founding Editor-in-Chief of the Institution of Engineering and Technology Signal Processing Journal. He is the Technical Program Cochair for IEEE Globecom 2010 to be held in Miami and served in the same role for the IEEE International Conference on Communications held in Glasgow, U.K., in June 2007. 